\title{
Microwave Properties of Ice-Phase Hydrometeors for Radar and Radiometers: Sensitivity to Model Assumptions
}

\author{
BenJAmin T. JOHnson AND GRAnT W. PETTY \\ Atmospheric and Oceanic Sciences, University of Wisconsin-Madison, Madison, Wisconsin \\ GAIL SKOFRONICK-JACKSON \\ NASA Goddard Space Flight Center, Greenbelt, Maryland
}

(Manuscript received 12 July 2011, in final form 9 July 2012)

\begin{abstract}
A simplified framework is presented for assessing the qualitative sensitivities of computed microwave properties, satellite brightness temperatures, and radar reflectivities to assumptions concerning the physical properties of ice-phase hydrometeors. Properties considered included the shape parameter $\mu$ of a gamma size distribution and the melted-equivalent mass median diameter $D_{0}$, the particle density, the dielectric mixing formula, and the choice of complex index of refraction for ice. These properties are examined for selected radiometer frequencies of $18.7,36.5,89.0$, and $150.0 \mathrm{GHz}$ and radar frequencies at 2.8, 13.4, 35.6, and 94.0 $\mathrm{GHz}$ - consistent with existing and planned remote sensing instruments. Passive and active microwave observables of ice particles are found to be extremely sensitive to the $D_{0}$ of the size distribution. Similar large sensitivities are found for variations in the ice volume fraction whenever the geometric mass median diameter exceeds approximately $1 / 8$ th of the wavelength. At $94 \mathrm{GHz}$ the two-way path-integrated attenuation is potentially large for dense/compact particles. The distribution parameter $\mu$ has a comparatively weak effect on any observable: less than $1-2 \mathrm{~K}$ in brightness temperature and a maximum of $2.7 \mathrm{~dB}$ ( $\mathrm{S}$ band only) in the effective radar reflectivity. Reversal of the roles of ice and air in the Maxwell Garnett dielectric mixing formula leads to a substantial change in both microwave brightness temperature $(\sim 10 \mathrm{~K})$ and radar reflectivity (approximately $2 \mathrm{~dB}$ across all frequencies). The choice of the complex index of refraction of ice can produce a $3 \%-4 \%$ change in the brightness temperature depression.
\end{abstract}

\section{Introduction}

Whether passive or active, microwave-based precipitation retrieval algorithms depend on accurate quantitative relationships between precipitating-cloud properties and microwave observables (Panegrossi et al. 1998; Petty 2001; Kulie et al. 2010). Because of a lack of direct knowledge of the physical and associated radiative properties of the precipitation particles, however, physical models used to derive these relationships have historically relied on a variety of subjective assumptions about the microphysical and microwave radiative transfer properties of individual hydrometeors within a cloud, for example, the shape, size, and composition of raindrops,

Corresponding author address: Benjamin Johnson, Goddard Space Flight Center, Code 613.1, 8800 Greenbelt Rd., Greenbelt, MD 20771.

E-mail: benjamin.t.johnson@nasa.gov snowflakes, aggregates, and graupel particles (Savage 1978; Smith et al. 1992; Matrosov 1998; Matrosov et al. 2005; Katsumata et al. 2000; Meneghini and Liao 2000; Zhao and Weng 2002; Donovan et al. 2004; Surussavadee and Staelin 2006; Casella et al. 2008). Moreover, even the three-dimensional distributions of bulk hydrometeor concentrations obtained from state-of-the-art cloud-resolving models appear to be alarmingly sensitive to the choice of microphysical scheme employed (Hashino and Tripoli 2007; Han et al. 2010; Wu and Petty 2010).

Figure 1 summarizes the chain of observations, models, and physical assumptions necessary to predict satelliteobserved microwave brightness temperatures or radar reflectivities associated with a particular precipitating-cloud system and/or surface precipitation rate. Inappropriate assumptions at any point in this chain introduce errors into model calculations of the microwave observables associated with a given environmental state. Whether these errors are tolerable depends on the magnitude and 
Inputs, Methods, \& Assumptions

\begin{tabular}{|l|}
\hline Particle physical description: \\
In situ observations and/or Models \\
\hline Simulated hydrometeor properties: \\
(1) Geometric Model (e.g., spheres) \\
(2) Dielectric composition \\
(e.g., dielectric mixing) \\
(3) Computational Method \\
(e.g., Mie Theory, DDA, etc.) \\
\hline
\end{tabular}

Distribution of mass:

(1) Particle Size Distribution (PSD)

(e.g., gamma, lognormal, etc.)

(2) Numerical Integration

Outputs

Individual hydrometeor physical properties:

(a) Size, (b) Shape, (c) Phase, (d) Composition

Individual hydrometeor microwave properties:

(a) Extinction, absorption, and scattering crosssections

(b) Scattering phase function

\section{PSD-integrated microwave properties:}

(a) Extinction coefficient per unit mass

(b) Radar backscatter per unit mass

(c) Single scatter albedo

(d) Scattering asymmetry parameter

Precipitating cloud simulation:

(1) Cloud Model: 1-D, 3-D

(2) Surface emissivity/reflectivity

(3) Radiative Transfer: 1-D, 3-D, polarized

\section{Cloud Physical Properties:}

(a) Spatial distribution of hydrometeors, water content

(b) Thermodynamics (e.g., Temperature) Microwave Observables:

(a) Passive microwave brightness temperature

(b) Attenuated radar backscatter

FIG. 1. The relationship between key model assumptions and computed microwave observables associated with a modeled precipitating-cloud system.

spectral dependence of the error and on whether the physical model is being used solely as theoretical guidance in algorithm design or is being depended upon to supply the quantitative basis [e.g., radar reflectivity-rainfall rate $(Z-R)$ relationship or brightness temperature database] for the retrievals.

This paper focuses solely on the sensitivity of computed passive and active microwave observables to plausible variations in the assumed properties of ice-phase hydrometeors in a precipitating-cloud system. Of potential concern are

(i) errors due to the assumed shapes of the individual ice particles (e.g., spheres vs more-realistic crystal or aggregate shapes),

(ii) errors due to the assumed size and/or density distributions of the particles within a volume of air, and

(iii) errors due to the dielectric constant assumed for pure ice and/or for subwavelength-scale mixtures of ice and air.

This paper is concerned strictly with the first and second categories of errors above. We note, however, that the microwave properties of realistic nonspherical snow and ice particles are the subject of ongoing investigation, as exemplified recently by Skofronick-Jackson et al. (2008), Noh et al. (2006), Hong (2007a,b), Kim et al. (2007, 2008),
Ishimoto (2008), Liu (2008), Kulie and Bennartz (2009), and Petty and Huang (2010). Nonspherical particles, while having apparently more "realistic" shapes, are still idealized models, the variations of which have not been fully examined in the literature. The authors have already made simulations of nonspherical particles in specific contexts—see, for example, Petty and Huang (2010) for $\mathrm{Ku}-$ and $\mathrm{Ka}-$ band radar properties.

Notwithstanding recent progress by the present authors and others listed above, published simulations of nonspherical particles are not extensive and varied enough to permit systematic sensitivity studies encompassing reasonable variations in particle size distributions (PSDs), particle densities, and other relevant properties. For example, Petty and Huang (2010) have found that a limited set of nonspherical particles is generally unable to reproduce the same frequencydependent scattering and extinction properties as spheres, no matter how one adjusts the densities. Of specific relevance to this work, however, is that computation using variable-density spheres "brackets" the currently computed nonspherical particle properties for a wide variety of frequencies, shapes, and sizes; in this sense, then, the study presented here provides a "worst case" scenario with respect to uncertainties arising out of variations in the simulated physical properties of hydrometeors. 
Furthermore, there is still considerable present-day modeling and algorithm-development work that relies on the soft-sphere model for lack of good practical alternatives. For example, the Goddard profiling algorithm (Kummerow et al. 1996) that is used in the Tropical Rainfall Measuring Mission, Advanced Microwave Scanning Radiometer for Earth Observing System (AMSR-E), and Global Precipitation Measurement (GPM) passive microwave retrieval algorithms continues to employ spherical particles in its brightness temperature computations.

To summarize, the "soft sphere" model discussed herein must be understood as providing useful guidance (as opposed to quantitative predictions) on the potential magnitude of uncertainties in forward radiative transfer models and retrieval algorithms. In the next section, we review the conceptual and mathematical framework for computing microwave and bulk physical properties from assumed distributions of hydrometeors, including specific assumptions about the hydrometeor dielectric model, bulk density, and size distribution. We specifically confine our attention to spherical ice particles having either a constant or a power-law bulk density and obeying a gamma PSD. We also discuss choices bearing on the assumed dielectric constant of an ice-air mixture, including both the dielectric constant of pure ice and the multiple possible "effective medium" approximationsin particular, those of Bruggeman (Bruggeman 1935) and the Maxwell Garnett methods (Maxwell Garnett 1904). We also consider two values for the complex dielectric constant of pure ice: one obtained from the widely used tabulation of Warren (1984, hereinafter W84) and the other from the recent update by Warren and Brandt (2008, hereinafter WB08). We conclude that section with a simplified two-stream radiative transfer framework for evaluating the approximate sensitivity of observed microwave brightness temperatures to variations in the hydrometeor model employed.

In section 3, we evaluate the sensitivities of bulk hydrometeor radiative properties, satellite microwave brightness temperatures, and radar backscatter at selected frequencies to plausible variations in the mass median diameter $D_{0}$, the size distribution shape parameter $\mu$, the particle density $\rho$, the choice of dielectric constant for pure ice, and the choice of dielectric mixing formula. For radiometric calculations, the chosen frequencies are 18.7, 36.5, 89.0, and $150 \mathrm{GHz}$. For radar backscatter calculations, we consider 2.8 , 13.4, 35.6, and $94 \mathrm{GHz}$. In the final section, we conclude with a summary of key results from section 3 and implications for the retrieval of precipitation and hydrometeor properties from passive and active microwave sensors.

\section{Physical and radiative properties}

Microwave radiances and reflectivities observed from either space- or surface-based sensors depend on the threedimensional distribution of local radiative transfer properties and temperature along and near the line of sight. The frequency-dependent and hydrometeor-dependent properties of interest here include the volume or mass extinction coefficient $\left(k_{e}\right.$ or $\left.\kappa_{e}\right)$, single-scattering albedo $\tilde{\omega}$, scattering asymmetry parameter $g$, and radar reflectivity $\eta$. These quantities are defined for a volume of air containing a suspension of particles; they are derived in turn from knowledge of the number and radiative cross sections and scattering phase functions of the individual particles in the volume.

In this section, we specify a model for the density of each particle as a function of particle diameter or mass. The density in turn maps to a microwave-frequencydependent dielectric constant according to either of two models for the dielectric constant for pure ice and one of three models for the dielectric constant of a mixture of ice and air. We calculate radiative cross sections for individual particles using standard Mie theory (Mie 1908) and then integrate over a specified PSD to obtain bulk radiative properties for an ensemble of particles. The details of these steps follow below. Because we are primarily interested in the sensitivity of radiative transfer calculations to variations in the physical properties of ice-phase hydrometeors, we do not consider the effects of absorption or emission by atmospheric gases or cloud liquid water, nor do we consider variations of surface emissivity.

\section{a. Particle density}

To assess the impact of ice-particle composition on the above-described radiative properties, we assume homogeneous dielectric spheres of mass $M$ and geometric diameter $D_{g}$ that are composed of a blend of ice and air-with $f_{\text {ice }}$ giving the corresponding volume fraction of ice such that $f_{\text {ice }}+f_{\text {air }}=1$. The bulk particle density is then

$$
\rho=6 M /\left(\pi D_{g}^{3}\right)=f_{\text {ice }} \rho_{i}=\left(D / D_{g}\right)^{3} \rho_{i},
$$

where we take $\rho_{i}=917 \mathrm{~kg} \mathrm{~m}^{-3}$ and neglect the mass of air (but not the volume) in these mixtures. Because of the multitude of morphological possibilities, the relationship between the geometric size of a snowflake and its effective density is not well characterized, but it is conventional to model the relationship as a power law:

$$
\rho\left(D_{g}\right)=a_{\rho} D_{g}^{-b_{\rho}}
$$


TABLE 1. Snow density parameters (centimeter-gram-second units) for Eq. (2) from Brown and Ruf (2007).

\begin{tabular}{lcc}
\hline \hline \multicolumn{1}{c}{ Source } & $a_{\rho}$ & $b_{\rho}$ \\
\hline Magono and Nakamura (1965) & 0.022 & 1.5 \\
Barthazy et al. (1998) & 0.018 & 0.8 \\
University of Wisconsin Nonhydrostatic & 0.015 & 0.6 \\
$\quad$ Modeling System (Tripoli 1992) & & \\
Locatelli and Hobbs (1974) & 0.015 & 1.18 \\
Mitchell et al. (1990) & 0.012 & 1.0 \\
\hline
\end{tabular}

We examine two cases that commonly occur in the literature: 1) the assumption of a constant density throughout the PSD $\left(b_{\rho}=0\right)$ and 2) a power-law densitysize relationship, of which five published examples are taken from Brown and Ruf (2007) and Szyrmer and Zawadzki (2010) and summarized in Table 1.

\section{b. Effective dielectric constant}

In the spherical-particle approximation, low-density ice particles are modeled as having a uniform dielectric constant appropriate to a particular mixture of ice and air. Even once this mixture has been specified, however, a surprising number of choices exist for computing the effective dielectric constant. One objective of this study is therefore to elucidate the practical sensitivity of microwave observables to the chosen mixing rule.

The two best-known mixing rules are the Bruggeman (Bruggeman 1935) or effective-medium approximation and the Maxwell Garnett (Maxwell Garnett 1904) or matrix-inclusion method. Both methods continue to be widely used in physical modeling of the microwave radiative properties of hydrometeors. These methods are summarized in Bohren and Huffman (1983). Casella et al. (2008), Petty and Huang (2010), and others have examined the consistency of these two common mixing rules with explicit discrete dipole approximation (DDA) calculations of mass-weighted extinction, scattering, and backscattering properties for idealized reduced-density spherical ice particles.

Among other things, Petty and Huang (2010) found that a generalized mixing rule falling roughly midway between the two cases gave the best fit to the DDAcalculated properties. In this study we extend their approach to incorporate a wider range of microwave frequencies and particle densities and to examine the sensitivity of variations in the previously described physical properties of hydrometeors on simulated/ idealized passive microwave brightness temperatures and radar reflectivities.

The Bruggeman formulation for a mixture of two components is

$$
\epsilon_{\mathrm{eff}}=\epsilon_{B}=\frac{1}{2}\left(\beta^{2}+2 \epsilon_{1} \epsilon_{2}\right)^{1 / 2}-\frac{1}{2} \beta,
$$

where

$$
\beta=\frac{1}{2}\left[\left(1-3 f_{2}\right) \epsilon_{2}+\left(1-3 f_{1}\right) \epsilon_{1}\right] .
$$

In Eq. (3), $\epsilon_{1}$ and $\epsilon_{2}$ are the dielectric constants of components 1 and 2 , respectively; $f_{1}$ and $f_{2}=1-f_{1}$ are the respective volume fractions; and $\epsilon_{B}$ is the effective dielectric constant of the mixture.

The Maxwell Garnett (MG) method was derived for a two-component mixture consisting of a matrix with dielectric constant $\epsilon_{m}$ and a fraction $f$ of small spherical inclusions with dielectric constant $\epsilon_{i}$. The effective dielectric constant according to this method is given by

$$
\epsilon_{\mathrm{eff}}=\epsilon_{\mathrm{MG}}=\epsilon_{m} \frac{\epsilon_{i}(1+2 f)-\epsilon_{m}(2 f-2)}{\epsilon_{m}(2+f)+\epsilon_{i}(1-f)} .
$$

Reversing the roles of $\epsilon_{i}, \epsilon_{m}$, and $f$ results in a different value for $\epsilon_{\mathrm{eff}}$ in the MG case.

Figure 2 illustrates the influence of ice-air volume fraction on the real and imaginary components of the dielectric function for the Bruggeman and two $\mathrm{MG}$ formulations. The top and bottom panels of Fig. 2 show the dielectric permittivities of ice from W84 and WB08, respectively; both are presented at $89 \mathrm{GHz}$ and a temperature of $266 \mathrm{~K}$. Each circle is labeled according to the volume fraction of ice.

It is apparent that at small volume fractions of ice the Bruggeman method is closer to MG- $\langle[\mathrm{I}] \mathrm{A}\rangle$, in which ice is the inclusion component. As the volume fraction of ice increases, however, the Bruggeman curve approaches the MG- $\langle[\mathrm{A}] \mathrm{I}\rangle$ curve, in which air is the inclusion component. The two MG methods effectively bracket the Bruggeman results (for all frequencies and ice-volume fractions tested; not shown), with Bruggeman being closest to the MG method where the volume fraction of the inclusion components is small (e.g., $f_{\text {incl }}<0.3$; Sihvola and Kong 1988, Lakhtakia and Shanker 1993, Mackay 2005). For particles having large size parameters, the size parameters of the individual inclusions may become unphysically large for the MG methods to be appropriate; therefore, additional care must be taken to ensure that this does not occur if one uses the MG method.

\section{c. Single-particle properties}

Any single hydrometeor can be characterized physically by its mass $M$, size, shape, and composition (e.g., fraction of ice vs liquid). Of these properties, only the mass and composition are unambiguous. Geometric 

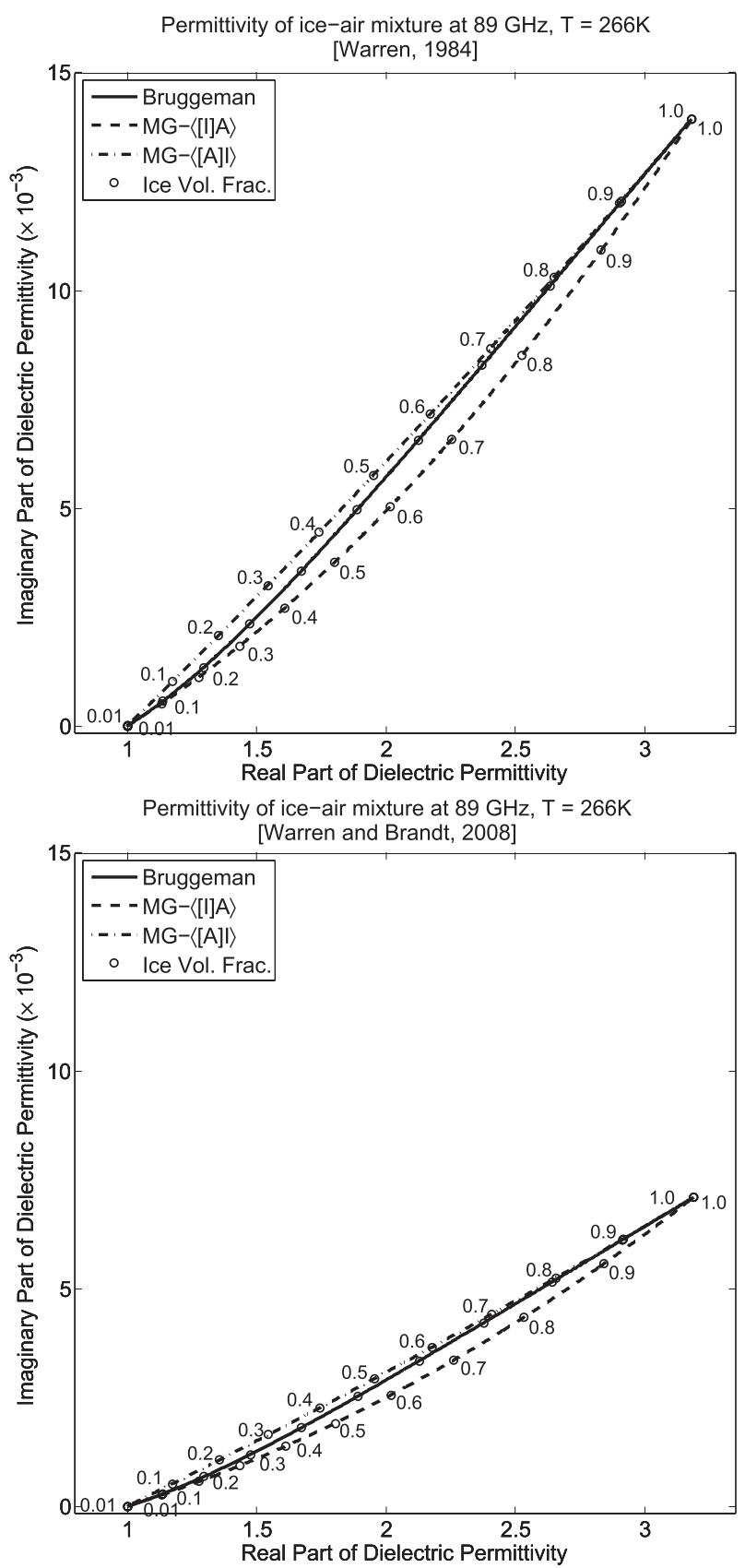

FIG. 2. Dielectric permittivity of ice-air material mixture at $89 \mathrm{GHz}$ and $266 \mathrm{~K}$ for ice volume fractions ranging from 0.01 to 1.0. The curves represent the Maxwell Garnett and Bruggeman formulations. Label MG-〈[A]I $\rangle$ indicates an ice matrix with air inclusions; MG- $\langle[\mathrm{I}] \mathrm{A}\rangle$ denotes the reverse. (top) The W84 permittivity; (bottom) the permittivity from WB08.

size, shape, and effective density are far more variable and are difficult even to define, especially in the case of complex snow aggregates (Petty and Huang 2010, 2011). One may also characterize the mass of an ice particle by its melted-equivalent spherical radius $D$, defined as

$$
D \equiv\left(\frac{6 M}{\pi \rho_{w}}\right)^{1 / 3},
$$

where $\rho_{w}$ is the density of water, taken to be $1000 \mathrm{~kg} \mathrm{~m}^{-3}$.

For a given microwave frequency, each particle also has an absorption cross section $\sigma_{a}$, scattering cross section $\sigma_{s}$, and scattering phase function $P(\Theta)$. For true nonspherical particles, these characteristics would exhibit a strong dependence on orientation; such effects fall outside the scope of this paper.

Given the geometric diameter $D_{g}$ and complex index of refraction $N$, where $N^{2}=\epsilon_{\text {eff }}$, the assumption of spherical shape allows us to use standard Mie theory (Mie 1908; Bohren and Huffman 1983) to compute the above properties and, from these, the extinction cross section:

$$
\sigma_{e} \equiv \sigma_{a}+\sigma_{s}
$$

and the radar backscatter cross section:

$$
\left.\sigma_{b} \equiv \sigma_{s} P(\Theta)\right|_{\Theta=\pi} .
$$

\section{d. Bulk microphysical properties}

Atmospheric remote sensing methods respond not to individual particles but rather to large numbers of suspended particles of different sizes, shapes, and orientations distributed more or less randomly throughout a substantial volume of the atmosphere. When dealing with single-phase ice particles, it is convenient (although by no means strictly accurate) to assume that the sole relevant independent variable is the particle mass $M$ or, alternatively, the melted-equivalent diameter $D$.

\section{SIZE DISTRIBUTION}

Following Ulbrich (1983), Brandes et al. (2004), and Heymsfield et al. (2005), and many others, we assume a three-parameter gamma PSD relative to the meltedequivalent diameter $D$ :

$$
n(D)=N_{0} D^{\mu} \exp (-\Lambda D)
$$

where $\Lambda$ is often referred to as the "slope parameter," $\mu$ is a "shape parameter," and $N_{0}$ controls the overall concentration of particles. ${ }^{1}$ It is important to note that

\footnotetext{
${ }^{1}$ As discussed by Petty and Huang (2011), a gamma distribution in $D$ is also a gamma distribution in $D_{g}$ only when density is constant. For the case in which the density is nonconstant and given by Eq. (2), the corresponding PSD expressed in $D_{g}$ is a four-parameter modified gamma distribution.
} 
the use of a continuous gamma distribution is not ideal for data for which the tails of the distribution may be underrepresented, such as in observational datasets. In such cases we recommend using a truncated gamma distribution (Ulbrich and Atlas 1998; Hogan et al. 2012). Here, we prefer the continuous gamma distribution for computational convenience.

The mass median melted-equivalent diameter $D_{0}$ is the characteristic size of the PSD that divides the total mass of the PSD into two equal parts and is given for the gamma distribution by

$$
D_{0} \approx \frac{\mu+3.67}{\Lambda} .
$$

The corresponding mass median geometric diameter $D_{0 g}$ is obtained with Eq. (1), and the ice water content is given by

$$
\mathcal{W}=\frac{\pi \rho_{w}}{6} \int_{0}^{\infty} D^{3} n(D) d D
$$

\section{e. Bulk radiative properties}

The radiative cross sections of individual particles are computed for the microwave frequencies of interest by using an updated version of the Mie routine published by Bohren and Huffman (1983). They are then summed over the PSD to yield the total cross sections per unit volume of air and related radiative properties. Of particular relevance are the volume extinction coefficient,

$$
k_{e} \equiv \int_{0}^{\infty} \sigma_{e}(D) n(D) d D,
$$

and the volume scattering coefficient $k_{s}$, defined similarly using the scattering cross section $\sigma_{s}(D)$. The ensemble single-scatter albedo is $\tilde{\omega} \equiv k_{s} / k_{e}$.

Here it is convenient to characterize extinction not in terms of the volume extinction $k_{e}\left(\mathrm{~m}^{-1}\right)$ but rather in terms of the mass extinction coefficient $\kappa_{e}\left(\mathrm{~m}^{2} \mathrm{~kg}^{-1}\right)$ :

$$
\kappa_{e} \equiv k_{e} / \mathcal{W}
$$

which represents the extinction cross section per unit mass along the line of sight. It has two advantages in the present context: first, it depends only on the shape of $n(D)$ and not on the magnitude; second, it allows optical path and transmission to be conveniently related to mass path along the line of sight rather than to the geometric distance.

For most radiative transfer calculations, we additionally require at least the asymmetry parameter $g$, which is defined as the first moment (with respect to $\cos \Theta$ ) of the ensemble scattering phase function $P(\Theta)$ (Bohren and Huffman 1983). The latter is in turn the scattering cross-section-weighted sum of the phase functions of the individual particles.

In the absence of attenuation, the effective radar reflectivity factor $Z_{e}$ is given by

$$
Z_{e} \equiv \frac{\lambda^{4}}{\pi^{5}\left|K_{w}\right|^{2}} \int_{0}^{\infty} \sigma_{b}(D) n(D) d D,
$$

where $\lambda$ is the radar wavelength and $\left|K_{w}\right|^{2}=0.93$ by convention.

\section{f. Radiative transfer}

The purpose of this paper is not to undertake accurate and detailed radiative transfer calculations but rather to evaluate relative sensitivities of microwave observables to varying assumptions about ice particle properties their PSDs. We may therefore take advantage of highly simplified treatments of the radiative transfer to characterize the expected magnitude of these responses.

\section{1) BRIGHTNESS TEMPERATURE}

The general theoretical basis for computing radiative transfer in a scattering and emitting medium is well known and need not be repeated here (Lenoble 1985; Liou 2002). For the purpose of sensitivity analysis, we introduce a simple analytic approximation that is based on a two-stream approximation (TSA) that has been found to be remarkably accurate at describing thermal emission from a plane-parallel slab viewed at the $\sim 55^{\circ}$ incidence typical of a satellite microwave imager. Weng and Grody (2000) and Liu and Weng (2002) have also discussed the utility of the TSA in a similar context; here, we develop expressions that starting with the TSA formulation as reviewed by Petty (2006).

For an isothermal finite slab of hydrometeors with temperature $T$ overlying a nonreflective surface with temperature $T_{s}$, the total optical thickness is $\tau=\kappa_{e} \mathcal{I}$, where $\mathcal{I}$ is the ice water path, and the upwelling microwave brightness temperature in the two-stream approximation is

$$
T_{B}=T_{s} t+(1-r-t) T,
$$

where $t$ is the total transmittance (direct plus diffuse) and $r$ is the reflectance. In the two-stream approximation, the reflectance and transmittance are in turn functions of $\tau$ and of the bulk single-scattering albedo $\tilde{\omega}$ and asymmetry parameter $g$ of the constituent particles within the slab: 


$$
\begin{aligned}
& r=\frac{r_{\infty}\left(e^{\Gamma \tau}-e^{-\Gamma \tau}\right)}{e^{\Gamma \tau}-r_{\infty}^{2} e^{-\Gamma \tau}} \text { and } \\
& t=\frac{1-r_{\infty}^{2}}{e^{\Gamma \tau}-r_{\infty}^{2} e^{-\Gamma \tau}},
\end{aligned}
$$

where

$$
\begin{gathered}
r_{\infty} \equiv \frac{\sqrt{1-g \tilde{\omega}}-\sqrt{1-g}}{\sqrt{1-g \tilde{\omega}}+\sqrt{1-g}} \text { and } \\
\Gamma \equiv 2[(1-\tilde{\omega} g)(1-\tilde{\omega})]^{1 / 2} .
\end{gathered}
$$

\section{2) EFFECTIVE RADAR REFLECTIVITY}

At the top of the slab, $Z_{e}$ is calculated using Eq. (13) applied to the assumed particle distribution. At the bottom of the slab, $Z_{e}$ is reduced by the two-way path attenuation. We ignore multiple scattering in these idealized sensitivity experiments, although we note that Matrosov et al. (2008) and Battaglia et al. (2008) have shown that multiple scattering (MS) can significantly enhance the observed reflectivity profiles at $94 \mathrm{GHz}$ above what single scattering alone would predict. At 13.4 and $35.6 \mathrm{GHz}$, the reflectivities appear to be relatively unaffected by MS in snowfall observations.

For nadir viewing by a satellite radar, the direct transmittance to the base of the slab is given by Beer's law: $\mathcal{T}=$ $\exp (-\tau)$ and may be expressed as a one-way path attenuation in decibels with $\mathcal{A}(\mathrm{dB})=-10 \log _{10} \mathcal{T}$. The twoway attenuation is, of course, 2 times this amount.

We may thus not only examine the effect of assumed particle properties on the intrinsic reflectivity $Z_{e}$ but also on the combined effect of changes in reflectivity and changes in two-way attenuation:

$$
Z_{e}^{\prime}(\mathrm{dB} Z)=Z_{e}(\mathrm{~dB} Z)-2 \mathcal{A}(\mathrm{dB}) .
$$

Unlike the case for radiometric quantities, which depend only on the path-integrated hydrometeor properties, reflectivity also depends on hydrometeor concentration per unit volume. Here, we assume a mass concentration $\mathcal{W}$ of $1 \mathrm{~g} \mathrm{~m}^{-3}$. For a typical mass-weighted fall speed of $1 \mathrm{~m} \mathrm{~s}^{-1}$, this value would imply a liquid-equivalent precipitation rate of $3.6 \mathrm{~mm} \mathrm{~h}^{-1}$.

\section{Sensitivity studies}

\section{a. Overview}

The variable parameters of $\rho$ [Eq. (1)], $\mu$ [Eq. (8)], and $D_{0}$ [Eq. (9)] fully characterize the composition and distribution of the ice-phase hydrometeor ensemble apart from the multiplicative constant $N_{0}$. In this paper, $N_{0}$ is required only for computing radar reflectivity $Z_{e}$. We set $N_{0}$ implicitly by specifying a fixed ice water content $\mathcal{W}$ of $1 \mathrm{~g} \mathrm{~m}^{-3}$, unless otherwise noted.

Choosing the microwave frequency $\nu$ and one of three candidate dielectric mixing formulas then allows all local microwave properties $\kappa_{e}, \tilde{\omega}, g$, and $Z_{e}$ to be computed, along with $T_{B}$ and $\mathcal{A}$ following from the relationships in section $2 \mathrm{f}$ and from the specified column ice water path $\mathcal{I}$ of $1 \mathrm{~kg} \mathrm{~m}^{-2}$.

We select four frequencies-18.7, 36.5, 89.0, and 150.0 $\mathrm{GHz}$ - that are representative of those found on current and planned microwave imagers. At each frequency, we examine the effects of varying $\rho, D_{0}, \mu$, and the mixing formula on the computed single-layer slab TSA $T_{B}$ values. We also performed calculations for $183.3 \mathrm{GHz}$ and found that it behaves qualitatively very much like $150 \mathrm{GHz}$; general conclusions in this paper concerning the latter frequency also apply to $183.3 \mathrm{GHz}$.

For our examination of the radar reflectivities $Z_{e}$ and $\mathcal{A}$, we select an additional four frequencies: $2.8,13.4$, 36.5, and $94.0 \mathrm{GHz}$. The first of these corresponds to the frequency utilized by the ground-based Weather Surveillance Radar-1988 Doppler (WSR-88D). The next two will be utilized by the GPM dual-frequency precipitation radar (DPR). The $94.0-\mathrm{GHz}$ frequency is utilized by the CloudSat radar.

For each of the above frequencies, we computed the relevant radiative properties by assuming spherical particles having either a constant density $\left(b_{\rho}=0.0\right)$ or a power-law density defined by Eq. (2) and Table 1 . We vary $\rho$ (through $a_{\rho}$ and $b_{\rho}$ ), $D_{0}$, and $\mu$ for each of the three dielectric mixing formulas and for both the WB08 and older W84 tabulations of the complex index of refraction of ice.

Representative values of all of these parameters were chosen to define a baseline against which all variations were compared. For this baseline, we assume a constant-density PSD with $f_{\text {ice }}=0.1\left(\rho \approx 91.7 \mathrm{~kg} \mathrm{~m}^{-3}\right)$, $D_{0}=1 \mathrm{~mm}$, and $\mu=0$ [i.e., $n(D)$ is exponential]; $\epsilon_{\text {ice }}$ is given by $\mathrm{WB} 08$, and the dielectric mixing rule is Bruggeman (B).

One parameter at a time was varied from the baseline value. In this case, $D_{0}$ was varied from $0.125 \mathrm{~mm}$, corresponding to fairly small cirrus particles, to $8.0 \mathrm{~mm}$, corresponding to large sleet, snow aggregates, or small hail. The ice fraction $f_{\text {ice }}$ was varied from 0.01 , corresponding to a very-low-density snow aggregate, to 1.0, corresponding to a solid ice pellet. The shape parameter $\mu$ was varied from -1 to 4 , encompassing the majority of empirically determined values for this parameter (e.g., Heymsfield 2003). Representative results are tabulated in Tables $2-4$, described in more detail below. 
TABLE 2. Local radiative transfer properties of varying ice particle distribution models for selected radiometer frequencies. Baseline parameter values are indicated in boldface. For ease of comparison, baseline results are repeated within each parameter grouping. The square brackets under "mixing rule" enclose the Maxwell Garnett inclusion component, either ice or air. Note that $\kappa_{e}$ has units of meters squared per kilogram.

\begin{tabular}{|c|c|c|c|c|c|c|c|c|c|}
\hline & \multicolumn{3}{|c|}{$36.5 \mathrm{GHz}$} & \multicolumn{3}{|c|}{$89.0 \mathrm{GHz}$} & \multicolumn{3}{|c|}{$150 \mathrm{GHz}$} \\
\hline & $\kappa_{e}$ & $\tilde{\omega}$ & $g$ & $\kappa_{e}$ & $\tilde{\omega}$ & $g$ & $\kappa_{e}$ & $\tilde{\omega}$ & $g$ \\
\hline \multicolumn{10}{|l|}{$f_{\text {ice }}($ constant $\rho)$} \\
\hline $0.01(0.009)$ & $0.80 \times 10^{-2}$ & 0.896 & 0.68 & $0.59 \times 10^{-1}$ & 0.917 & 0.90 & 0.18 & 0.921 & 0.96 \\
\hline $0.03(0.028)$ & $0.13 \times 10^{-1}$ & 0.937 & 0.52 & 0.11 & 0.955 & 0.84 & 0.35 & 0.958 & 0.92 \\
\hline $0.1(0.092)$ & $0.23 \times 10^{-1}$ & 0.960 & 0.33 & 0.24 & 0.976 & 0.73 & 0.78 & 0.978 & 0.86 \\
\hline $0.3(0.28)$ & $0.38 \times 10^{-1}$ & 0.971 & 0.21 & 0.51 & 0.985 & 0.59 & 1.7 & 0.986 & 0.76 \\
\hline $1.0(0.92)$ & $0.48 \times 10^{-1}$ & 0.980 & 0.15 & 1.00 & 0.990 & 0.44 & 3.1 & 0.986 & 0.53 \\
\hline \multicolumn{10}{|l|}{$a_{\rho}, b_{\rho}$ [Eq. (2)] } \\
\hline $0.022,1.50$ & $0.75 \times 10^{-1}$ & 0.964 & 0.26 & 1.12 & 0.983 & 0.50 & 4.86 & 0.985 & 0.65 \\
\hline $0.012,1.00$ & $0.26 \times 10^{-1}$ & 0.961 & 0.32 & 0.27 & 0.977 & 0.72 & 0.83 & 0.979 & 0.85 \\
\hline $0.015,1.18$ & $0.31 \times 10^{-1}$ & 0.960 & 0.32 & 0.34 & 0.977 & 0.68 & 1.16 & 0.980 & 0.80 \\
\hline $0.018,0.80$ & $0.25 \times 10^{-1}$ & 0.961 & 0.32 & 0.26 & 0.977 & 0.72 & 0.87 & 0.979 & 0.85 \\
\hline $0.012,0.60$ & $0.24 \times 10^{-1}$ & 0.961 & 0.32 & 0.25 & 0.977 & 0.72 & 0.83 & 0.979 & 0.86 \\
\hline \multicolumn{10}{|l|}{$D_{0}(\mathrm{~mm})$} \\
\hline 0.125 & $0.99 \times 10^{-3}$ & 0.091 & 0.01 & $0.83 \times 10^{-2}$ & 0.356 & 0.04 & $0.36 \times 10^{-1}$ & 0.571 & 0.12 \\
\hline 0.25 & $0.16 \times 10^{-2}$ & 0.433 & 0.03 & $0.24 \times 10^{-1}$ & 0.774 & 0.16 & 0.11 & 0.860 & 0.35 \\
\hline 0.5 & $0.56 \times 10^{-2}$ & 0.836 & 0.11 & $0.85 \times 10^{-1}$ & 0.934 & 0.43 & 0.33 & 0.950 & 0.66 \\
\hline 1.0 & $0.23 \times 10^{-1}$ & 0.960 & 0.33 & 0.24 & 0.976 & 0.73 & 0.78 & 0.978 & 0.86 \\
\hline 2.0 & $0.73 \times 10^{-1}$ & 0.987 & 0.65 & 0.56 & 0.989 & 0.89 & 1.58 & 0.989 & 0.95 \\
\hline 4.0 & 0.18 & 0.995 & 0.86 & 1.08 & 0.994 & 0.96 & 2.48 & 0.993 & 0.97 \\
\hline 8.0 & 0.37 & 0.997 & 0.94 & 1.54 & 0.996 & 0.97 & 2.39 & 0.993 & 0.89 \\
\hline \multicolumn{10}{|l|}{$\mu$} \\
\hline-1.0 & $0.26 \times 10^{-1}$ & 0.963 & 0.39 & 0.25 & 0.977 & 0.75 & 0.81 & 0.979 & 0.87 \\
\hline 0.0 & $0.23 \times 10^{-1}$ & 0.960 & 0.33 & 0.24 & 0.976 & 0.73 & 0.78 & 0.978 & 0.86 \\
\hline 1.0 & $0.22 \times 10^{-1}$ & 0.957 & 0.30 & 0.23 & 0.975 & 0.71 & 0.77 & 0.978 & 0.86 \\
\hline 2.0 & $0.21 \times 10^{-1}$ & 0.956 & 0.27 & 0.23 & 0.975 & 0.70 & 0.76 & 0.978 & 0.85 \\
\hline 3.0 & $0.20 \times 10^{-1}$ & 0.954 & 0.25 & 0.22 & 0.974 & 0.69 & 0.75 & 0.978 & 0.85 \\
\hline 4.0 & $0.20 \times 10^{-1}$ & 0.953 & 0.23 & 0.22 & 0.974 & 0.69 & 0.75 & 0.977 & 0.85 \\
\hline \multicolumn{10}{|l|}{$\epsilon_{\text {ice }}$} \\
\hline WB08 & $0.23 \times 10^{-1}$ & 0.960 & 0.33 & 0.24 & 0.976 & 0.73 & 0.78 & 0.978 & 0.86 \\
\hline W84 & $0.25 \times 10^{-1}$ & 0.894 & 0.33 & 0.24 & 0.953 & 0.73 & 0.78 & 0.966 & 0.86 \\
\hline \multicolumn{10}{|l|}{ Mixing rule } \\
\hline $\mathrm{MG}-\langle[\mathrm{I}] \mathrm{A}\rangle$ & $0.22 \times 10^{-1}$ & 0.961 & 0.33 & 0.22 & 0.977 & 0.73 & 0.73 & 0.979 & 0.86 \\
\hline Bruggeman & $0.23 \times 10^{-1}$ & 0.960 & 0.33 & 0.24 & 0.976 & 0.73 & 0.78 & 0.978 & 0.86 \\
\hline $\mathrm{MG}-\langle[\mathrm{A}] \mathrm{I}\rangle$ & $0.37 \times 10^{-1}$ & 0.955 & 0.34 & 0.38 & 0.973 & 0.73 & 1.2 & 0.975 & 0.86 \\
\hline
\end{tabular}

\section{b. Effects on local optical properties}

A number of generalizations may be made about the effects of model parameters on the fundamental microwave radiative properties $\kappa_{e}, \tilde{\omega}$, and $g$ shown in Table 2 . Note that the $18.7-\mathrm{GHz}$ results discussed below are not shown in Table 2.

First, it is apparent that, for fixed $D_{0}=1 \mathrm{~mm}$, the assumed particle density has a profound effect on the mass extinction coefficient $\kappa_{e}$ at all frequencies. The higher the frequency is, the more dramatic is the effect. In particular, the difference in mass extinction coefficient between $f_{\text {ice }}=0.01$ and $f_{\text {ice }}=1$ (constant-density PSDs) ranges from slightly more than a factor of 2 at $18.7 \mathrm{GHz}$ to a factor of 17 at $150 \mathrm{GHz}$.

For the variable-density relationships, when $b_{\rho}$ is greater than unity, there is a significantly stronger sensitivity in $\kappa_{e}$ than for when $b_{\rho}$ is less than unity; over the five examples, we find as much as a factor-of-6 difference at $150 \mathrm{GHz}$. At all frequencies, the setting of $a_{\rho}=0.022$ and $b_{\rho}=1.50$ produces larger $\kappa_{e}$ values than do even solid ice spheres.

Higher-density spheres have higher single-scatter albedos and smaller values of $g$. The latter effect is expected because high density implies a smaller geometric dimension relative to the wavelength and thus more symmetric scattering. In particular, in the Rayleigh limit $\left(D_{0 g} \ll \lambda\right)$, we expect $g=0$. At the other extreme, lowdensity spheres exhibit very strong forward scattering, with $g$ approaching the theoretical limit of 1 at the highest frequency.

Not surprising is that variations in $D_{0}$ also strongly affect microwave properties, with larger particle masses being associated with sharply higher extinction per unit 
mass. The sensitivity is largest at the lowest frequencies. A factor-of- 4 change in $D_{0}$ (from 0.5 to $2.0 \mathrm{~mm}$ ) gives rise to a factor-of-19 difference in $\kappa_{e}$ at $18.7 \mathrm{GHz}$ but only a factor-of-5 difference at $150 \mathrm{GHz}$. Again, this spectral dependence is not unexpected, because the transition from lower to higher frequency is associated with a move farther away from the Rayleigh regime, with its $D^{6}$ scattering dependence, into the Mie regime, where extinction efficiencies are more nearly constant.

The distribution shape parameter $\mu$ turns out to have only a very subtle effect on radiative properties. For fixed $D_{0}$ and fixed $\mathcal{W}$, increasing values of $\mu$ act to sharpen and narrow the particle mass distribution $D^{3} n(D)$ - that is, there is an increase in the mass contribution of particles with sizes near $D_{0}$ with compensating decreases at the large and small ends of the distribution. At the lowest frequency, $\kappa_{e}$ is approximately $50 \%$ larger for $\mu=-1$ than it is for $\mu=4$.

The choice of W84 or WB08 to specify the complex index refraction of ice primarily affects the imaginary part, which is roughly a factor-of- 2 smaller in the newer compilation. This difference gives rise to a roughly proportional change in the absorption coefficient of the particles and thus in the single-scatter coalbedo $1-\tilde{\omega}$. Where the latter is moderately large (primarily at the lowest frequencies), there is also a noticeable increase in the overall extinction coefficient.

Furthermore, we consider the choice of the dielectric mixing formula. The baseline choice is the Bruggeman formulation (B). The two alternatives considered are the Maxwell Garnett formulation with ice as the inclusion $(\mathrm{MG}-\langle[\mathrm{I}] \mathrm{A}\rangle)$ or with ice as the matrix $(\mathrm{MG}-\langle[\mathrm{A}] \mathrm{I}\rangle)$. We see that $B$ and $M G-\langle[\mathrm{I}] \mathrm{A}\rangle$ yield very similar results overall while markedly higher absorption and overall extinction are associated with MG- $\langle[\mathrm{A}] \mathrm{I}\rangle$, particularly at the lowest frequencies. This effect has been noted in previous studies, and we corroborate it here as well.

In view of the very strong dependence of $\kappa_{e}$ on both $D_{0}$ and $\rho$, it is worth considering the full range of combinations of these two variables as in Fig. 3. These contour plots reveal that the maximum in $\kappa_{e}$ is achieved for very dense particles with $D_{0} \approx \lambda$. From this maximum, $\kappa_{e}$ falls off by at least two orders of magnitude for $D_{0}<0.5 \mathrm{~mm}$. As frequency increases, so does the sensitivity to particle density, which we suspect will be true with nonspherical particles as well, providing a basis for determining particle composition/density if the characteristic sizes of the particles are known (e.g., from dualwavelength radar observations).

In Fig. 4a, we have plotted $\kappa_{e}$ versus $D_{0}$ for the five selected size-density relationships (points). Also included, for reference, is $\kappa_{e}$ for selected constant-density values (curves). It is evident that the variable-density quantities exhibit striking differences relative to the constant-density curves, especially in the two cases in which $b_{\rho}$ is greater than unity. This point is especially relevant to researchers who employ constant-density spheres in that they may be unnecessarily biasing their results toward lower extinction values for a given $D_{0}$ (or ice water content) value.

\section{1) Results For Unit ICE WATER PATH}

We have previously stated that the two-stream approximation, while novel, is sufficient to qualitatively explore the brightness temperature response to variations of the physical properties of ice hydrometeors present in the atmosphere. With this context in mind, we now consider the combined effect of $\kappa_{e}, \tilde{\omega}$, and $g$ on characteristic brightness temperature depressions relative to the $273-\mathrm{K}$ background, using the aforementioned $\mathcal{I}=1 \mathrm{~kg} \mathrm{~m}^{-2}$ as the benchmark. For other values of $\mathcal{I}$, brightness temperatures can readily be computed from Table 2 and Eq. (14); in general, however, one can expect the brightness temperature depression to be roughly proportional to $\mathcal{I}$ at least down to several tens of kelvin below $273 \mathrm{~K}$.

In addition to the tabulated values in Table 3, we examine the response to joint variations in $D_{0}$ and $\rho$ in Figs. 4-6. In considering these together, we note the following behavior:

1) For fixed ice water path, the brightness temperature depression is very sensitive to both $D_{0}$ and $\rho$. The minimum $T_{B}$ (maximum scattering-induced depression) occurs for the variable-density formulation of Magono and Nakamura (1965) in Fig. 4c (only $150 \mathrm{GHz}$ is shown). For constant-density PSDs, $D_{0} \approx 0.6 \lambda$ (Fig. 5), and the strongest $T_{B}$ depressions occur at the highest-density particles (solid spheres).

2) As expected from the results in Table 2, the parameter $\mu$ has a nearly negligible effect on $T_{B}$ for modest ice water path, amounting to a maximum spread of $1.6 \mathrm{~K}$ at $150 \mathrm{GHz}$ when $D_{0}=1 \mathrm{~mm}$ and $\rho=0.092 \mathrm{~g} \mathrm{~cm}^{-3}\left(f_{\text {ice }}=0.1\right)$. We examined the response for the full range of $\rho$ and $D_{0}$ (not shown) and found that $\mu$ only begins to play a significant role in $T_{B}$ at $150 \mathrm{GHz}$ for $D_{0}>4 \mathrm{~mm}$, in which case $T_{B}$ may be as much as $10-20 \mathrm{~K}$ greater for $\mu=4$ than it is for $\mu=-1$.

3) The consequences of choosing W84 or WB08 for the index of refraction are potentially substantial, especially when considering the selected mass-size relationships. While the maximum response for baseline $\left(\rho=0.092 \mathrm{~g} \mathrm{~cm}^{-3}\right.$ and $\left.D_{0}=1 \mathrm{~mm}\right)$ is only a few tenths of a degree for any frequency, the full range 
a) $18.7 \mathrm{GHz}$

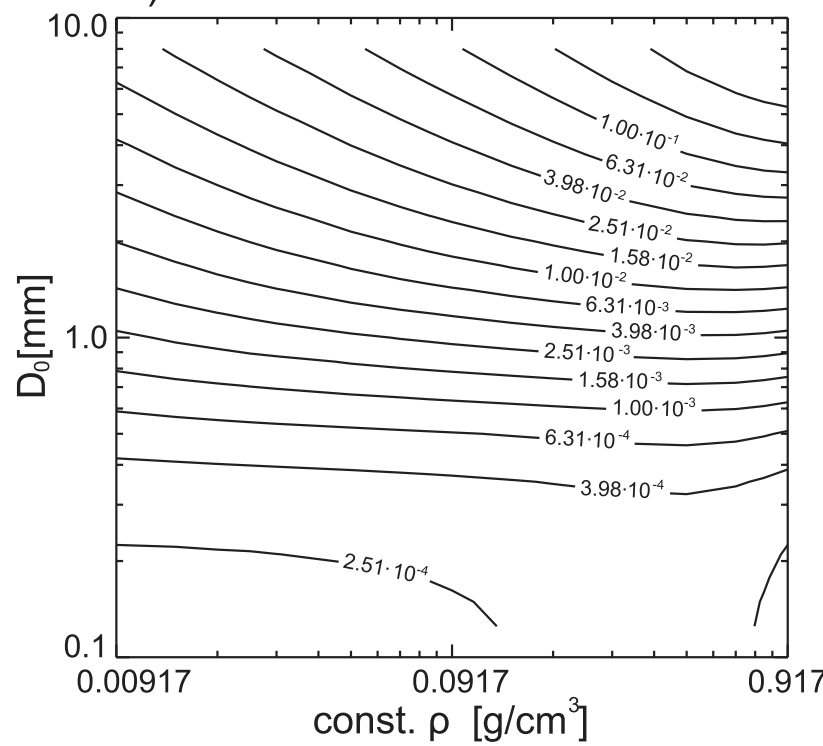

c) $89.0 \mathrm{GHz}$

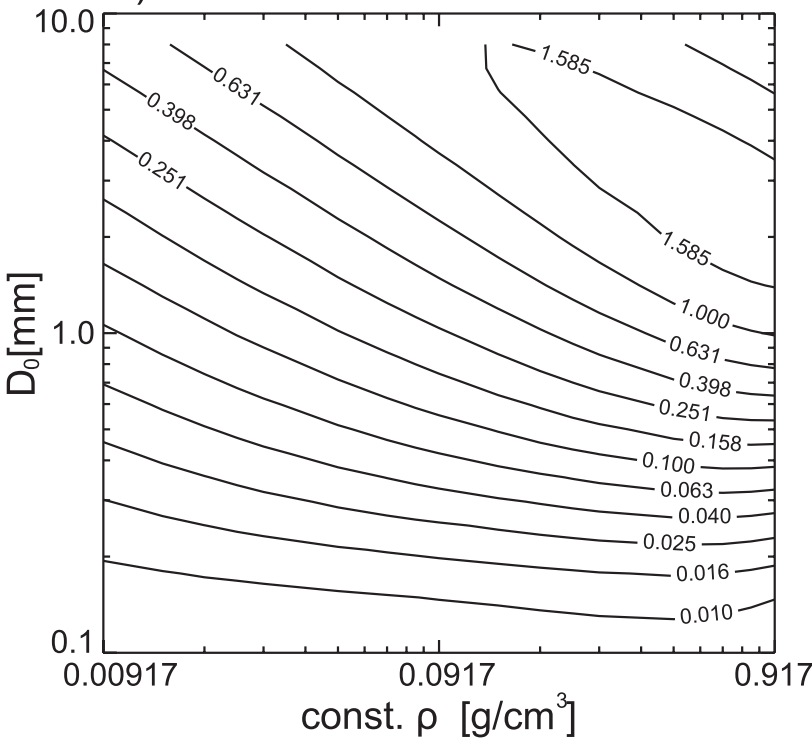

Mass Extinction Coefficient [ $\mathrm{m}^{2} / \mathrm{kg}$ ]

b) $36.5 \mathrm{GHz}$

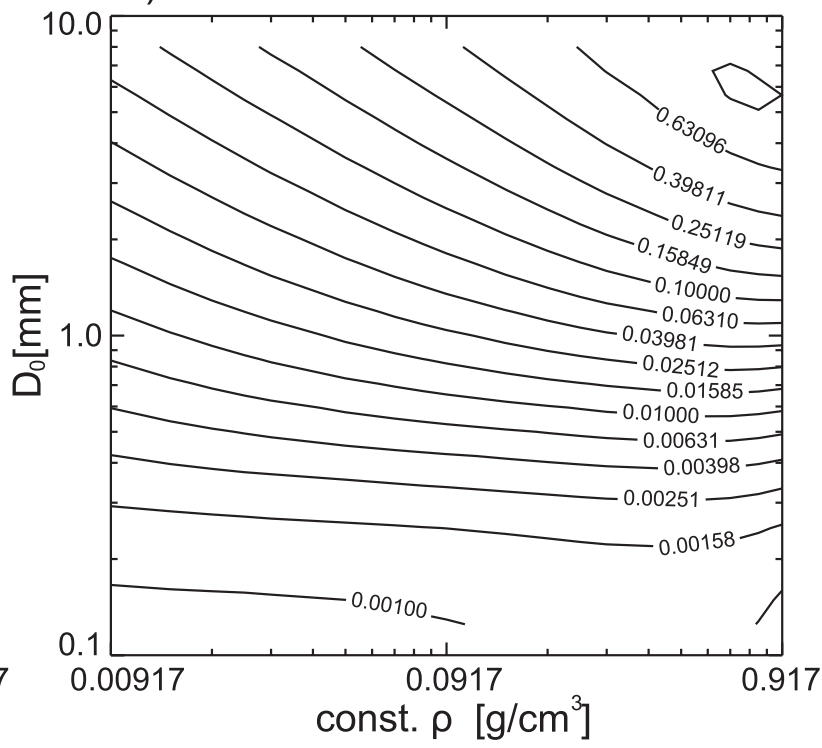

d) $150.0 \mathrm{GHz}$

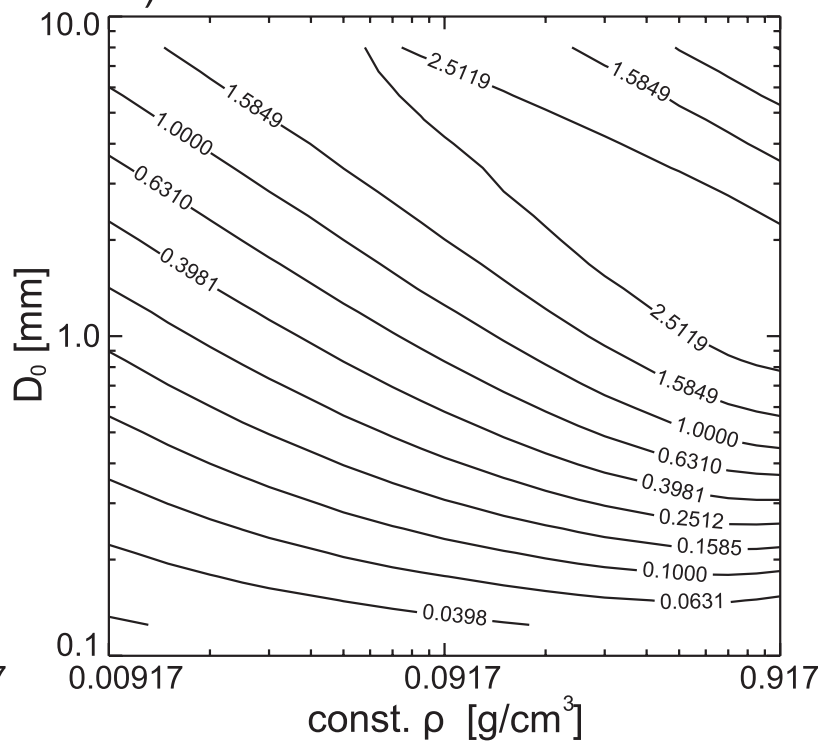

FIG. 3. The mass extinction coefficient $\kappa_{e}$ of ice obeying an exponential size distribution depicted as a function of the particle density $\rho$ and melted equivalent mass median diameter $D_{0}$ (contour values are logarithmically spaced) for (a) 18.7 , (b) 36.5 , (c) 89.0 , and (d) $150.0 \mathrm{GHz}$.

of $\rho$ and $D_{0}$ has a maximum sensitivity of as much as $14^{\circ}$ for $89 \mathrm{GHz}$ and $27^{\circ}$ at $150 \mathrm{GHz}$-mainly for denser particles (see Fig. 6).

4) The choice of dielectric mixing formulations has a minor impact at lower frequencies. However, $T_{B}$ depressions at 89 and $150 \mathrm{GHz}$ are "artificially" enhanced when using MG- $\langle[\mathrm{A}] \mathrm{I}\rangle$ because of the asymmetric treatment of ice as the matrix material.

\section{2) Two-Channel RATIO of $T_{B}$ DEPRESSIONS}

For any given microwave frequency, the scatteringinduced brightness temperature depression $S$, defined in the context of this analysis as

$$
S \equiv 273-T_{B}
$$




\section{Spherical Particle Properties @ 150 GHz constant density (solid lines) \& 5 size-density relationships (points)}
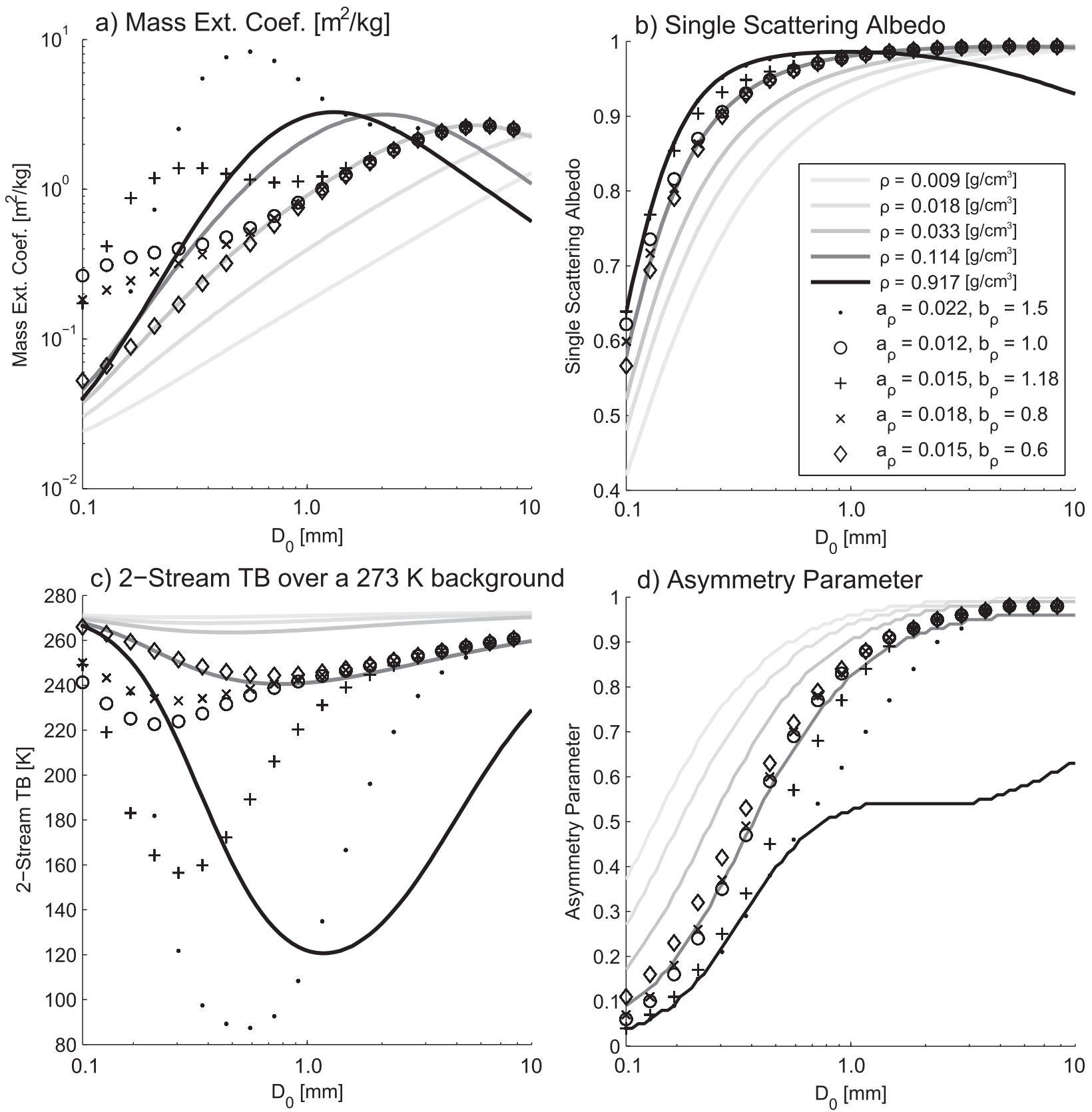

FIG. 4. Distribution-averaged properties at $150 \mathrm{GHz}$ : (a) mass extinction coefficient, (b) single-scattering albedo, (c) the two-stream brightness temperature associated with an isothermal $(T=266 \mathrm{~K})$ layer of exponentially distributed ice particles $\left(\mathcal{I}=1 \mathrm{~kg} \mathrm{~m}{ }^{-2}\right)$ and melted equivalent diameter $D_{0}$ overlying a black surface having temperature $273 \mathrm{~K}$, and $(\mathrm{d})$ the scattering asymmetry parameter. Solid lines are associated with constant density throughout the PSD; points indicate variable size-density relationships [Eq. (2)].

is clearly a sensitive function of three variables: $\mathcal{I}, D_{0}$, and $\rho$. It can be shown that, for fixed frequency, particle size, and density, $S$ is approximately proportional to $\mathcal{I}$ as long as the ice-layer optical thickness is less than about unity. But since $D_{0}$ and $\rho$ are unknown and strongly affect $S$ as well, single-channel retrievals of $\mathcal{I}$ are unlikely to be meaningful.

By using multiple radiometer frequencies, one might hope to isolate the effects of $\mathcal{I}, D_{0}$, and $\rho$, allowing 
TABLE 3. Radiometric response to ice particle distribution parameters as shown by the brightness temperature $T_{B}(\mathrm{~K})$ for an ice water path $\mathcal{I}$ of $1 \mathrm{~kg} \mathrm{~m}^{-2}$. Ice-layer temperature is $266 \mathrm{~K}$; background brightness temperature is $273 \mathrm{~K}$. Baseline parameter values are indicated in boldface. For ease of comparison, baseline results are repeated within each parameter grouping.

\begin{tabular}{|c|c|c|c|c|}
\hline & $18.7 \mathrm{GHz}$ & $36.5 \mathrm{GHz}$ & $89.0 \mathrm{GHz}$ & $150 \mathrm{GHz}$ \\
\hline \multicolumn{5}{|l|}{$f_{\text {ice }}($ constant $\rho)$} \\
\hline $0.01(0.009)$ & 272.8 & 272.4 & 271.5 & 271.1 \\
\hline $0.03(0.028)$ & 272.6 & 271.4 & 268.5 & 265.9 \\
\hline $0.1(0.092)$ & 272.4 & 269.0 & 256.9 & 247.3 \\
\hline $0.3(0.28)$ & 272.2 & 265.3 & 226.9 & 197.5 \\
\hline $1.0(0.92)$ & 272.2 & 262.5 & 177.2 & 122.0 \\
\hline \multicolumn{5}{|l|}{$a_{\rho}, b_{\rho}[$ Eq. (2)] } \\
\hline $0.022,1.50$ & 271.2 & 259.3 & 178.5 & 117.2 \\
\hline $0.012,1.00$ & 272.3 & 268.5 & 254.2 & 242.6 \\
\hline $0.015,1.18$ & 272.2 & 267.6 & 247.5 & 224.8 \\
\hline $0.018,0.80$ & 272.3 & 268.6 & 254.5 & 243.4 \\
\hline $0.015,0.60$ & 272.4 & 268.7 & 255.3 & 245.1 \\
\hline \multicolumn{5}{|l|}{$D_{0}(\mathrm{~mm})$} \\
\hline 0.125 & 273.0 & 273.0 & 272.2 & 268.1 \\
\hline 0.25 & 273.0 & 272.8 & 268.8 & 257.0 \\
\hline 0.5 & 272.9 & 271.9 & 261.2 & 247.3 \\
\hline 1.0 & 272.4 & 269.0 & 256.9 & 247.3 \\
\hline 2.0 & 270.9 & 266.3 & 257.4 & 253.4 \\
\hline 4.0 & 269.5 & 266.3 & 261.8 & 254.6 \\
\hline 8.0 & 269.4 & 267.1 & 261.0 & 218.0 \\
\hline \multicolumn{5}{|l|}{$\mu$} \\
\hline-1.0 & 272.3 & 268.9 & 257.3 & 248.2 \\
\hline 0.0 & 272.4 & 269.0 & 256.9 & 247.2 \\
\hline 1.0 & 272.4 & 269.0 & 256.2 & 247.6 \\
\hline 2.0 & 272.5 & 269.0 & 256.0 & 246.3 \\
\hline 3.0 & 272.5 & 269.1 & 255.7 & 246.5 \\
\hline 4.0 & 272.5 & 269.1 & 255.9 & 246.6 \\
\hline \multicolumn{5}{|l|}{$\epsilon_{\text {ice }}$} \\
\hline WB08 & 272.4 & 269.0 & 256.9 & 247.3 \\
\hline W84 & 272.4 & 269.0 & 257.1 & 247.7 \\
\hline \multicolumn{5}{|l|}{ Mixing rule } \\
\hline $\mathrm{MG}-\langle[\mathrm{I}] \mathrm{A}\rangle$ & 272.4 & 269.2 & 258.1 & 248.7 \\
\hline Bruggeman & 272.4 & 269.0 & 256.9 & 247.3 \\
\hline $\mathrm{MG}-\langle[\mathrm{A}] \mathrm{I}\rangle$ & 272.0 & 266.8 & 248.6 & 236.2 \\
\hline
\end{tabular}

reasonably unambiguous retrievals of each (Weng and Grody 2000; Zhao and Weng 2002). The success of this approach depends on there being a significant spectral dependence of $S$ on $D_{0}$ and/or $\rho$. One measure of such a dependence is the ratio of scattering depressions $S_{1} / S_{2}$ for pairs of frequencies $\nu_{1}$ and $\nu_{2}$.

Figures $7 \mathrm{a}$ and $7 \mathrm{~b}$ depict contours of the depression ratios $S_{89} / S_{36}$ while Figs. $7 \mathrm{c}$ and $7 \mathrm{~d}$ show the contours for $S_{150} / S_{89}$. These results indicate that the depression ratio is a sensitive indicator of $D_{0}$. In particular, for all but the lowest-density particles, Fig. 7a shows that measurements of $S_{89} / S_{36}$ allow $D_{0}$ to be estimated fairly unambiguously between approximately 0.1 and $3 \mathrm{~mm}$ while Fig. 7c shows that $S_{150} / S_{89}$ resolves variations of $D_{0}$ in the range of $0.2-1.0 \mathrm{~mm}$.
The sensitivity to particle density $\rho$ is very low for ratios of either pair of frequencies. Yet we have seen in Fig. 5 that, for fixed $D_{0}$, the magnitude of the response of any of these three frequencies depends strongly on both $\mathcal{I}$ and $\rho$. The variable-density relationships (Figs. $7 \mathrm{~b}$ and $7 d)$ illustrate a more complex story. It is evident that given a ratio of $S_{89} / S_{36}$ one can find a range of sensitivity to particle sizes between 0.1 and $7 \mathrm{~mm}$, depending on which density model is assumed. Comparing this with $S_{150} / S_{89}$ allows for the selection of which of the two possible $D_{0}$ values is appropriate (i.e., with which side of the peak on is concerned), as long as one sticks with a given size-density relationship (or constant density value). The implication is that, although retrievals of $D_{0}$ may be straightforward, the separation of $\mathcal{I}$ from $\rho$ may not be. Direct retrievals of $\mathcal{I}$ will most likely depend on reasonably confident specifications of the effective ice particle density $\rho$.

\section{c. Effects on observed radar backscatter}

We now turn our attention to the effects of ice-model parameters on backscattered microwave radiation as observed by ground-based or space-based radars. There are two major components to this response: the intrinsic effective radar reflectivity $Z_{e}$ of the distribution and the modification of this apparent reflectivity due to attenuation by the intervening medium. For a given nonzero mass path, it is possible for variations in model parameters to alter both $Z_{e}$ and the two-way path attenuation $(2 \mathcal{A})$ in ways that either reinforce or partially cancel each other.

For our analysis, we choose four representative frequencies corresponding to the ground-based WSR-88Ds $(2.8 \mathrm{GHz})$, the two channels of the spaceborne GPM DPR (13.4 and $36.5 \mathrm{GHz}$ ), and the CloudSat profiling radar $(94 \mathrm{GHz})$. Sample results are presented in Table 4. As before, we also examine the full range of combinations of $D_{0}$ and $\rho$ for selected frequencies in Figs. 8 and 9.

Seven general results were obtained:

1) For 13.4, 35.6, and $94 \mathrm{GHz}$, the effective reflectivity per unit mass is dominated by Rayleigh scattering and is nearly independent of $\rho$ for $D_{0 g}$ (and not $D_{0}$ ) of less than approximately 3,2 , and $0.5 \mathrm{~mm}$, respectively. For $2.8 \mathrm{GHz}$ (not shown), the corresponding threshold is approximately $14 \mathrm{~mm}$. Within this regime, single-frequency estimates of ice or snow water content require only accurate knowledge of the melted-equivalent mass median diameter $D_{0}$, assuming an ideally sensitive radar.

2) For $D_{0 g}$ that is much larger than the above thresholds, $Z_{e}$ depends equally strongly on both $D_{0}$ and $\rho$.

3) For 13.4 and $36.5 \mathrm{GHz}$, attenuation per unit mass is much less than $1 \mathrm{~dB}$ except for large $D_{0}$ combined 


\section{TB $[\mathrm{K}]$ for $1 \mathrm{~kg} / \mathrm{m}^{2}$ IWP over $273 \mathrm{~K}$ background}

a) $18.7 \mathrm{GHz}$

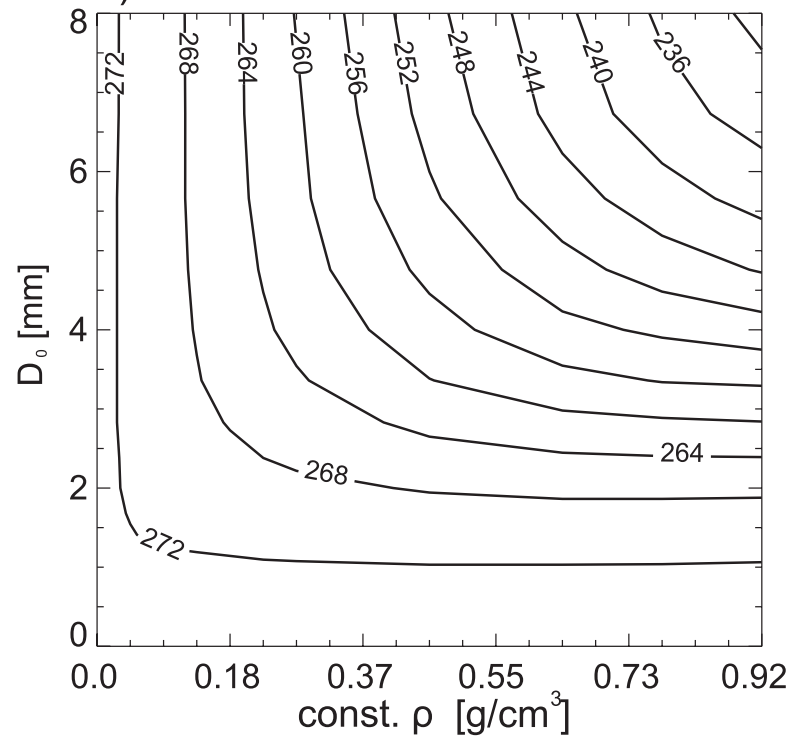

c) $89.0 \mathrm{GHz}$

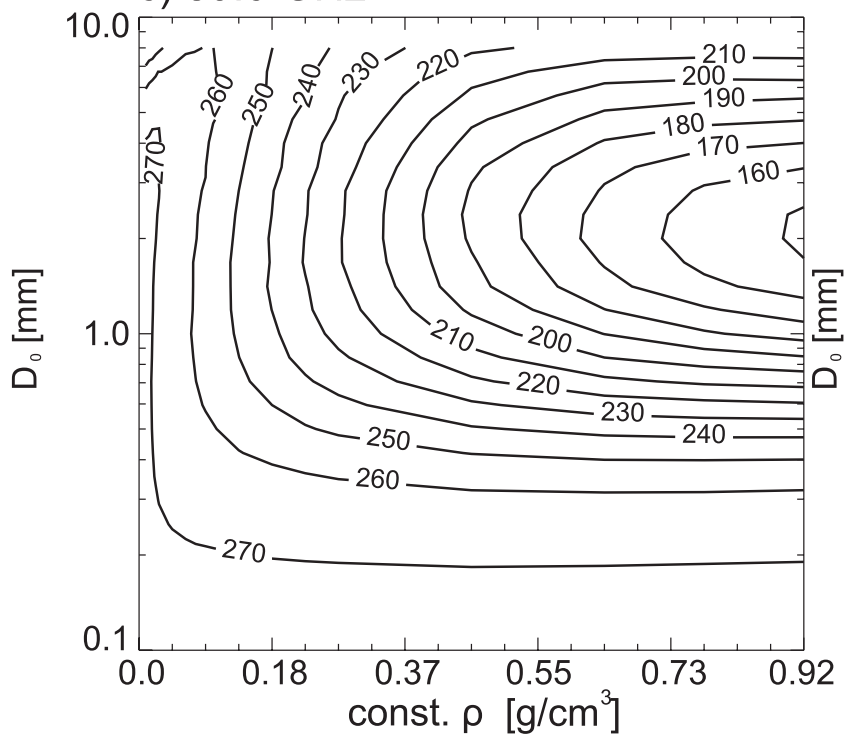

b) $36.5 \mathrm{GHz}$

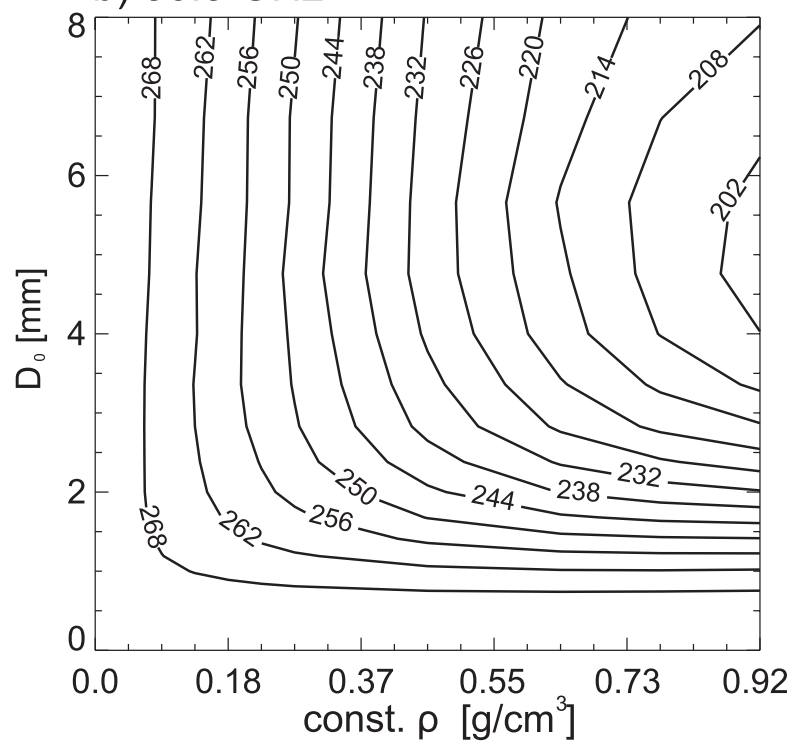

d) $150.0 \mathrm{GHz}$

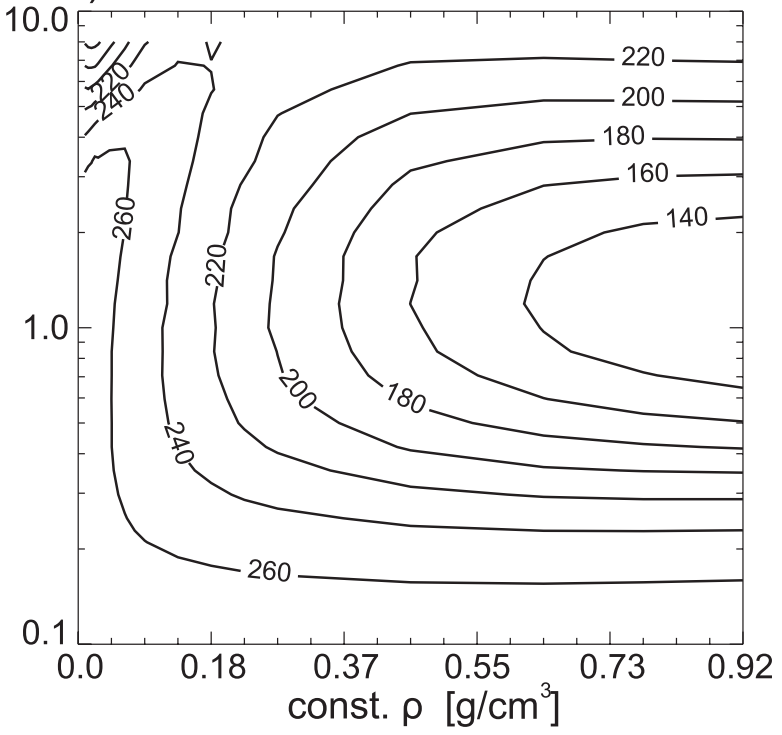

FIG. 5. The brightness temperature associated with an isothermal $(T=266 \mathrm{~K})$ layer of exponentially distributed ice particles $(\mathcal{I}=$ $1 \mathrm{~kg} \mathrm{~m}^{-2}$ ) with density $\rho$ and melted equivalent diameter $D_{0}$ overlying a black surface having temperature $273 \mathrm{~K}:$ (a) 18.7 , (b) 36.5 , (c) 89 , and (d) $150 \mathrm{GHz}$. Note the use of a linear axis for $D_{0}$ in (a) and (b) and a logarithmic axis in (c) and (d).

with moderate to high density, in which case it can be as much as $6 \mathrm{~dB}\left(\mathrm{~kg} \mathrm{~m}^{-2}\right)^{-1}$ at $35.6 \mathrm{GHz}$.

4) For $D_{0}=1 \mathrm{~mm}$ and $\rho=0.092 \mathrm{~g} \mathrm{~cm}^{-3}\left(f_{\text {ice }}=0.1\right)$, the parameter $\mu$ has no more than a few tenths of a reflectivity decibel effect on either $Z_{e}$ or $\mathcal{A}$ at any frequency. Only at large sizes (not shown) does the effect of $\mu$ start to be significant, and even then it is minor relative to the effect of variations in $D_{0}$ and $\rho$.
5) The choice of WB08 or W84 for the dielectric constant makes no significant difference for radar reflectivity or attenuation.

6) As before, the use of the MG- $\langle[\mathrm{A}] \mathrm{I}\rangle$ mixing model enhances scattering by a couple of decibels relative to Bruggeman (B) or $\mathrm{MG}-\langle[\mathrm{I}] \mathrm{A}\rangle$. At $94 \mathrm{GHz}$, increased attenuation over $1 \mathrm{~kg} \mathrm{~m}^{-2}$ is also significant. 
a) $89.0 \mathrm{GHz}$

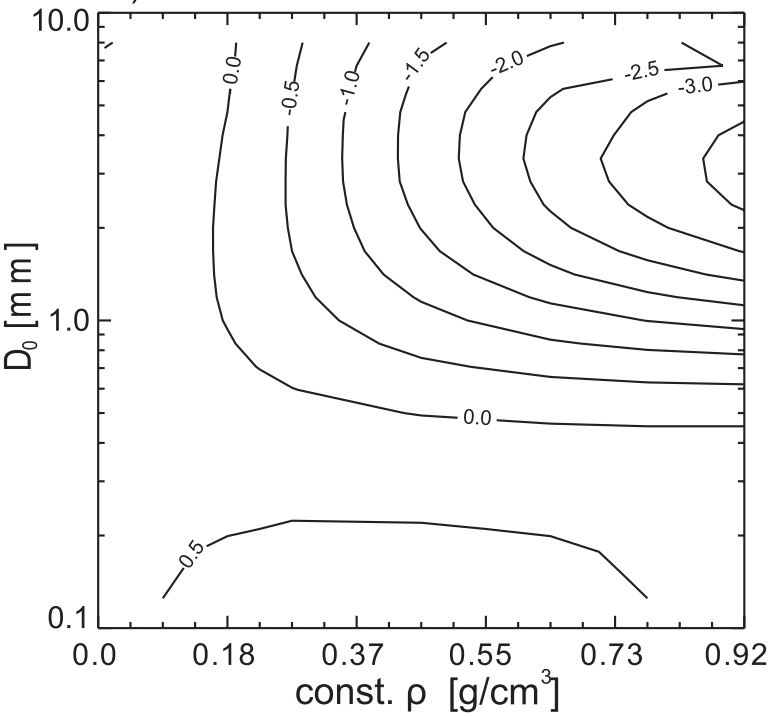

c) $150.0 \mathrm{GHz}$

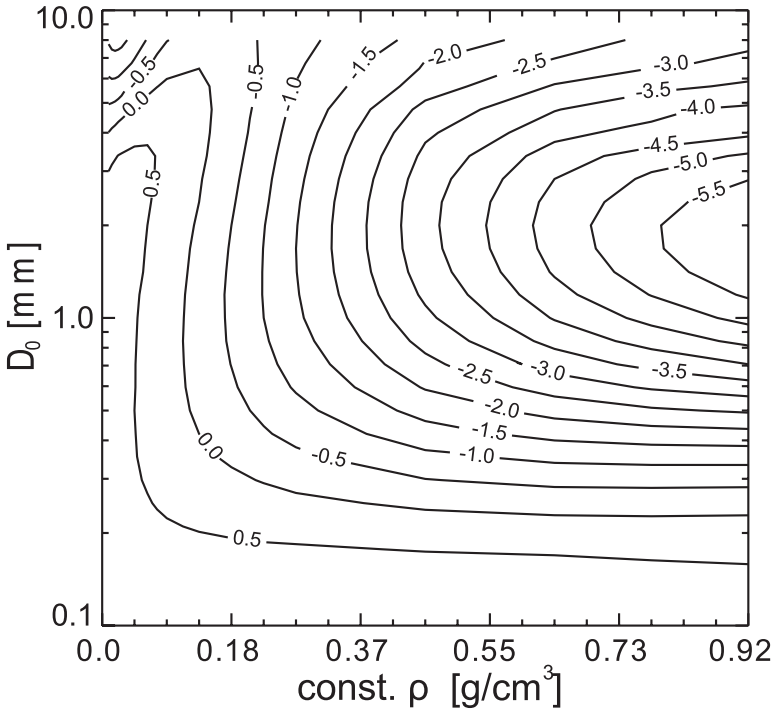

$T_{B}(W B 08)-T_{B}(W 84)[K]$

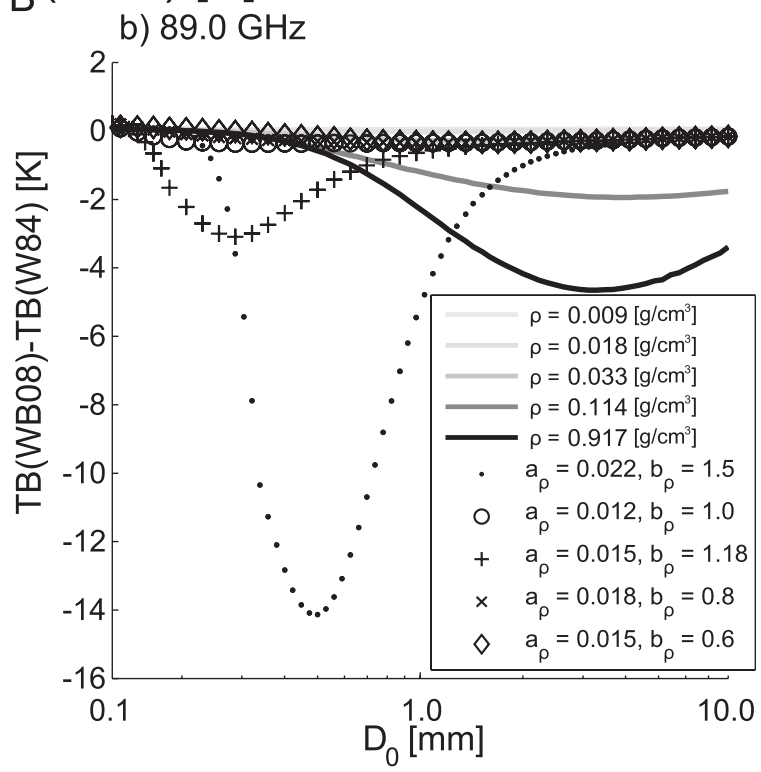

d) $150.0 \mathrm{GHz}$

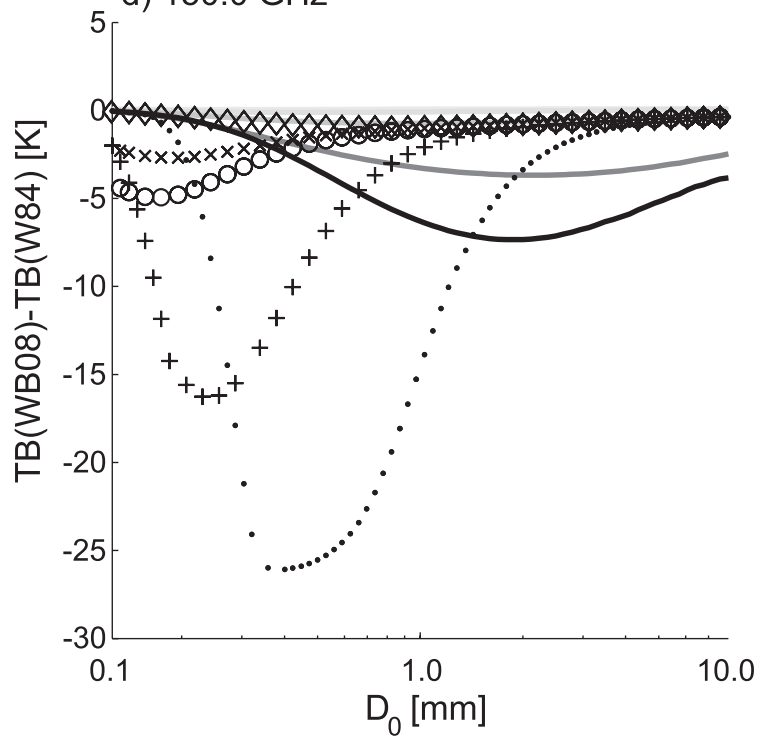

FIG. 6. Similar to Fig. 5, but contours depict the change in brightness temperature that results from choosing the W84 or WB08 values for the dielectric constant of ice: (a) 89 and (c) $150 \mathrm{GHz}$. Also shown are scatterplots of the same brightness temperature difference vs $D_{0}$ for various mass-density relationships at (b) 89 and (d) $150 \mathrm{GHz}$.

7) At the CloudSat frequency of $94 \mathrm{GHz}$, radar backscatter and attenuation are both very strongly sensitive to both $D_{0}$ and $\rho$ for all but the smallest values of $D_{0 g}$. Moreover, nearly solid ice particles with $D_{0}$ of 2-3 mm-representative of large, dense graupel or sleet-exhibit two-way path attenuation as high as $17 \mathrm{~dB}\left(\mathrm{~kg} \mathrm{~m}^{-2}\right)^{-1}$. These results would seem to preclude any snow water content retrieval from CloudSat alone without accurate independent knowledge of both $\rho$ and $D_{0}$, or constraints imposed by collocated passive microwave observations at $89 \mathrm{GHz}$ and higher (e.g., from the scattering ratios from Fig. 7).

\section{Conclusions}

We have presented a simplified framework for assessing the qualitative sensitivities of computed microwave properties, satellite brightness temperatures, and radar reflectivities to assumptions about the physical properties of ice-phase hydrometeors. Properties considered included 


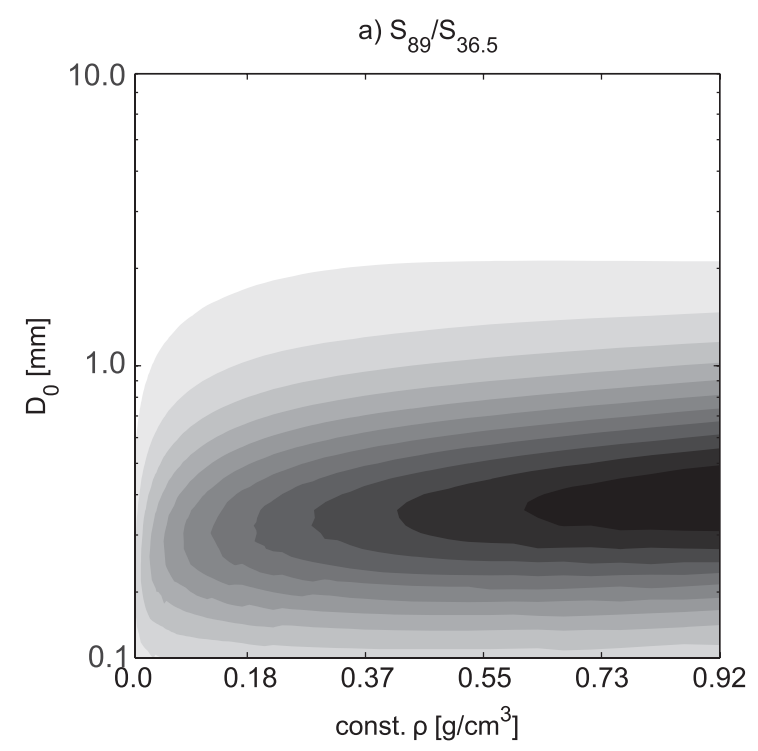

c) $\mathrm{S}_{150} / \mathrm{S}_{89}$

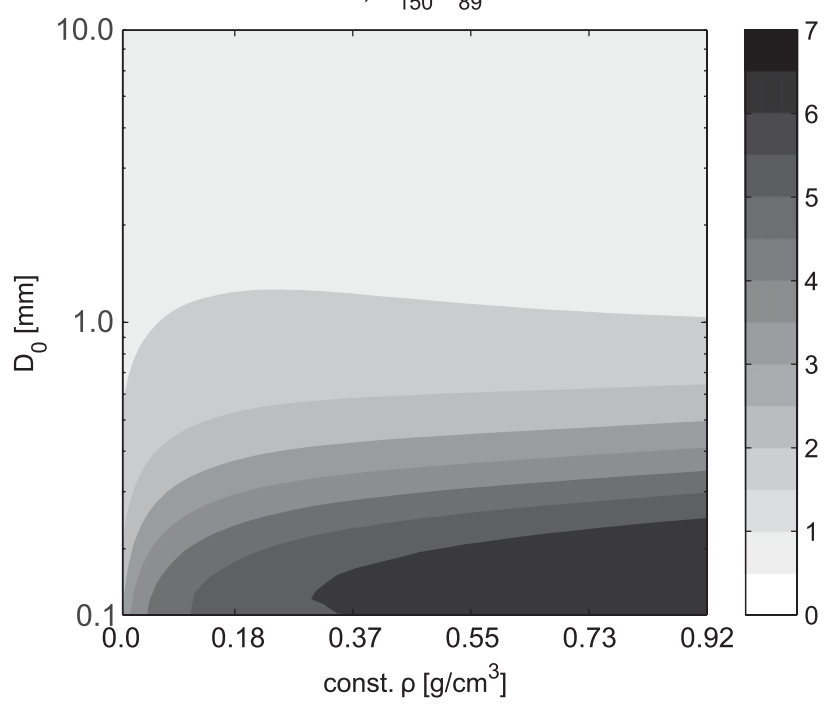

b) $\mathrm{S}_{89} / \mathrm{S}_{36.5}$
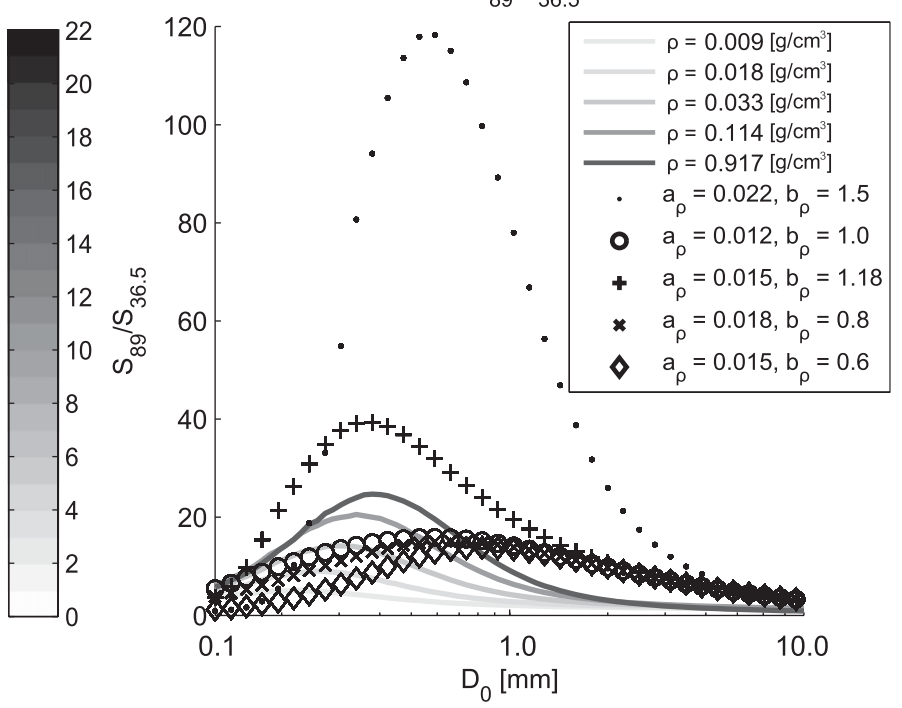

d) $\mathrm{S}_{150} / \mathrm{S}_{89}$

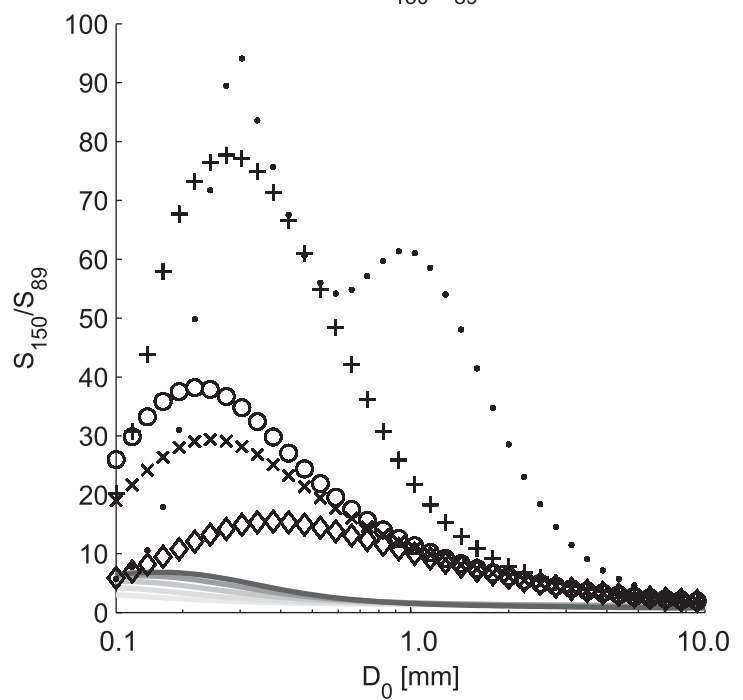

FIG. 7. The two-frequency ratio of brightness temperature depression (relative to a 273-K background) for the finite ice layer described in Fig. 5. (a) The 89-GHz depression divided by the 36-GHz depression for constant-density PSDs. (b) As in (a), but for variable-density PSDs [with constant curves (solid lines)] for comparison. (c) The 150-GHz depression divided by the 89-GHz depression. (d) As in (c), but for variable-density PSDs.

the shape and slope parameters of a gamma size distribution, particle shape and density, dielectric mixing formula, and the choice of complex index of refraction for ice.

On the basis of our analysis, we find that all passive and active microwave observables of ice particles are extremely sensitive to the melted-equivalent mass median diameter $D_{0}$ of the size distribution, all other parameters being held constant. Similar large sensitivities are found for the ice fraction $f_{\text {ice }}$ whenever the geometric mass median diameter $D_{0 g}$ exceeds approximately $1 / 8$ th of the wavelength. Uncertainties in the correct specification of $D_{0}$ and/or $f_{\text {ice }}$ will likely dominate the errors associated with both forward model calculations and physical retrievals from satellite microwave observations.

By contrast, the distribution shape parameter $\mu$ has a surprisingly weak effect on any observable, amounting to no more than a $1-2-\mathrm{K}$ variation in high-frequency brightness temperature for a $1 \mathrm{~kg} \mathrm{~m}^{-2}$ ice water path. For the radar backscatter at 13.4 and $35.6 \mathrm{GHz}$, the effect of varying $\mu$ from -1 to 4 was slightly more pronounced, yielding up to a $2.7-\mathrm{dB}$ difference in the backscatter ratio for a distribution with $D_{0}=1 \mathrm{~mm}$. 
TABLE 4. Results for selected radar frequencies. Baseline parameter values are indicated in boldface. Attenuation $\mathcal{A}$ is expressed as decibels per kilogram per meter squared of ice water path.

\begin{tabular}{|c|c|c|c|c|c|c|c|}
\hline & \multirow{2}{*}{$\begin{array}{l}2.8 \mathrm{GHz} \\
Z_{e}(\mathrm{dBZ})\end{array}$} & \multicolumn{2}{|c|}{$13.4 \mathrm{GHz}$} & \multicolumn{2}{|c|}{$35.6 \mathrm{GHz}$} & \multicolumn{2}{|c|}{$94.0 \mathrm{GHz}$} \\
\hline & & $Z_{e}(\mathrm{~dB} Z)$ & $2 \mathcal{A}(\mathrm{dB})$ & $Z_{e}(\mathrm{~dB} Z)$ & $2 \mathcal{A}(\mathrm{dB})$ & $Z_{e}(\mathrm{dBZ})$ & $2 \mathcal{A}(\mathrm{dB})$ \\
\hline \multicolumn{8}{|l|}{$f_{\text {ice }}($ constant $\rho)$} \\
\hline $0.01(0.0092)$ & 30.0 & 25.1 & 0.00 & 12.4 & 0.07 & -4.8 & 0.58 \\
\hline $0.03(0.028)$ & 30.2 & 27.6 & 0.01 & 18.2 & 0.11 & 1.9 & 1.13 \\
\hline $0.1(0.092)$ & 30.5 & 29.3 & 0.01 & 23.5 & 0.19 & 9.5 & 2.35 \\
\hline $0.3(0.28)$ & 30.8 & 30.4 & 0.01 & 27.3 & 0.30 & 16.5 & 5.04 \\
\hline $1.0(0.92)$ & 30.2 & 30.1 & 0.01 & 29.0 & 0.37 & 21.6 & 10.42 \\
\hline \multicolumn{8}{|l|}{$a_{\rho}, b_{\rho}[$ Eq. (2)] } \\
\hline $0.022,1.50$ & 30.6 & 29.6 & 0.02 & 25.4 & 0.60 & 18.4 & 11.40 \\
\hline $0.012,1.00$ & 30.5 & 29.4 & 0.01 & 23.9 & 0.21 & 10.2 & 2.65 \\
\hline $0.015,1.18$ & 30.5 & 29.4 & 0.01 & 24.0 & 0.25 & 12.4 & 3.35 \\
\hline $0.018,0.80$ & 30.5 & 29.4 & 0.01 & 23.9 & 0.20 & 10.2 & 2.61 \\
\hline $0.012,0.60$ & 30.5 & 29.4 & 0.01 & 23.9 & 0.20 & 10.0 & 2.51 \\
\hline \multicolumn{8}{|l|}{$D_{0}(\mathrm{~mm})$} \\
\hline 0.125 & 3.4 & 3.4 & 0.00 & 3.3 & 0.01 & 2.5 & 0.08 \\
\hline 0.25 & 12.4 & 12.4 & 0.00 & 11.9 & 0.01 & 8.9 & 0.25 \\
\hline 0.5 & 21.5 & 21.2 & 0.00 & 19.4 & 0.04 & 11.2 & 0.85 \\
\hline 1.0 & 30.5 & 29.3 & 0.01 & 23.5 & 0.19 & 9.5 & 2.37 \\
\hline 2.0 & 39.3 & 35.1 & 0.04 & 23.1 & 0.60 & 6.5 & 5.43 \\
\hline 4.0 & 47.7 & 36.5 & 0.14 & 20.4 & 1.49 & 3.4 & 10.34 \\
\hline 8.0 & 54.3 & 34.4 & 0.39 & 17.2 & 3.08 & 0.8 & 14.24 \\
\hline \multicolumn{8}{|l|}{$\mu$} \\
\hline-1.0 & 31.6 & 29.9 & 0.01 & 23.2 & 0.21 & 9.4 & 2.48 \\
\hline 0.0 & 30.5 & 29.3 & 0.01 & 23.5 & 0.19 & 9.5 & 2.37 \\
\hline 1.0 & 29.8 & 28.8 & 0.01 & 23.7 & 0.18 & 9.5 & 2.30 \\
\hline 2.0 & 29.3 & 28.5 & 0.01 & 23.9 & 0.17 & 9.4 & 2.27 \\
\hline 3.0 & 28.9 & 28.2 & 0.01 & 24.0 & 0.16 & 9.3 & 2.23 \\
\hline 4.0 & 28.7 & 28.0 & 0.01 & 24.0 & 0.16 & 9.3 & 2.21 \\
\hline \multicolumn{8}{|l|}{$\epsilon_{\text {ice }}$} \\
\hline WB08 & 30.5 & 29.3 & 0.01 & 23.5 & 0.19 & 9.5 & 2.35 \\
\hline W84 & 30.5 & 29.3 & 0.01 & 23.5 & 0.20 & 9.5 & 2.43 \\
\hline \multicolumn{8}{|l|}{ Mixing rule } \\
\hline $\mathrm{MG}-\langle[\mathrm{I}] \mathrm{A}\rangle$ & 30.2 & 29.0 & 0.01 & 23.2 & 0.17 & 9.2 & 2.17 \\
\hline Bruggeman & 30.5 & 29.3 & 0.01 & 23.5 & 0.19 & 9.5 & 2.35 \\
\hline $\mathrm{MG}-\langle[\mathrm{A}] \mathrm{I}\rangle$ & 32.4 & 31.2 & 0.01 & 25.5 & 0.30 & 11.6 & 3.73 \\
\hline
\end{tabular}

The choice of the Bruggeman versus Maxwell Garnett formulations for the effective dielectric constant of an ice-air mixture proved to be of at most very minor importance $(<1.5 \mathrm{~K}$ and $<0.3 \mathrm{~dB})$, provided that one uses Maxwell Garnett with ice as the inclusion in an air matrix. Reversal of the roles of ice and air leads to a significant change in both microwave brightness temperature $(\sim 10 \mathrm{~K})$ and radar reflectivity $(2 \mathrm{~dB})$.

For particle sizes and densities associated with strong scattering-based brightness temperature depressions at higher microwave frequencies (e.g., 89 and $150 \mathrm{GHz}$ ), switching from the older Warren (1984) index of refraction to the newer Warren and Brandt (2008) produces a roughly $3 \%-4 \%$ increase in the magnitude of the scattering-induced depression when constant-density spheres are assumed. If one includes the Magono and Nakamura size-density relationship, then the difference between WB08 and W84 is as much as $14 \mathrm{~K}$ at $89 \mathrm{GHz}$ and $27 \mathrm{~K}$ at $150 \mathrm{GHz}$. The choice has negligible effect on radar backscatter, however.

The ratio of scattering-induced brightness temperature depressions for 36 and $89 \mathrm{GHz}$ and for 89 and $150 \mathrm{GHz}$ is found to be sensitive primarily to the melted-equivalent mass median diameter $D_{0}$, with only very weak sensitivity to the density. These ratios, combined with the sensitivity of the GPM DPR to geometric mass median diameter $D_{0 g}$, suggest that the DPR can directly complement GPM microwave imager (GMI) observations of the scattering depression to infer both particle size and density for a broad range of characteristic particle sizes, assuming one adopts one of the several available particle-density models.

For the higher-frequency radars, the CloudSat radar at $94 \mathrm{GHz}$ is extremely sensitive to both $D_{0}$ and $\rho$, and so retrievals of ice water content and/or precipitation ratio from this instrument alone would be extremely sensitive 


\section{$Z_{\text {eff }}[d B Z]$}

\section{a) $13.4 \mathrm{GHz}$}

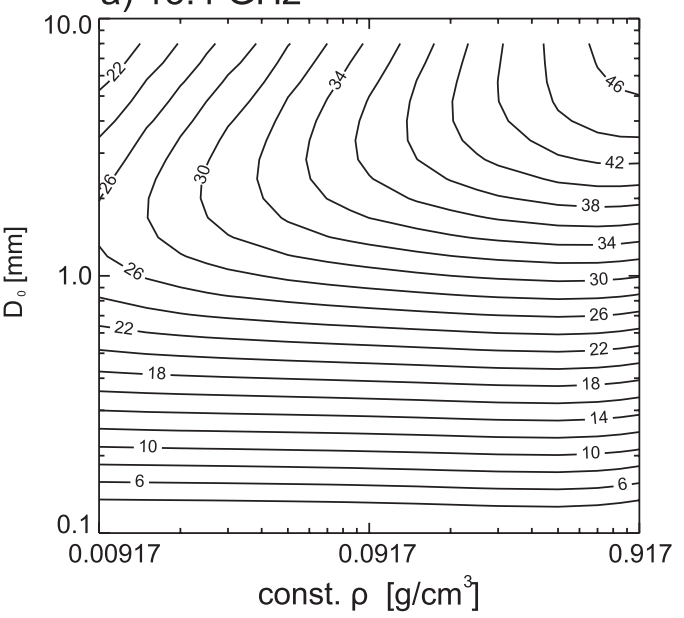

c) $35.6 \mathrm{GHz}$

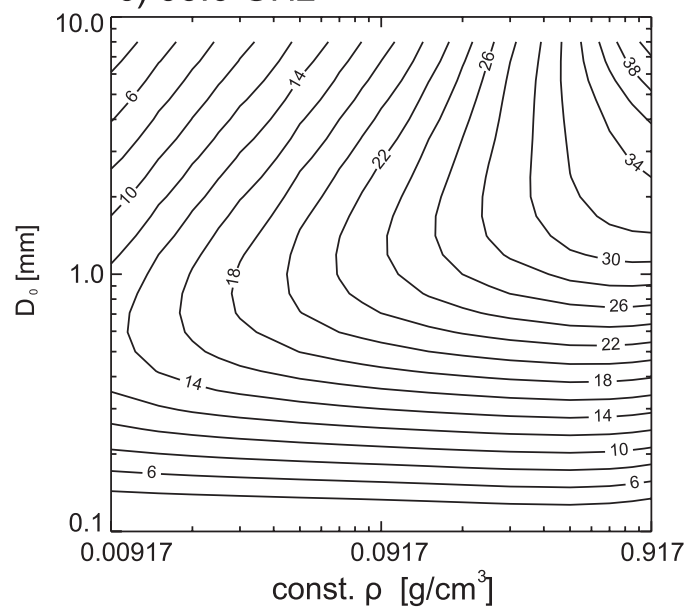

e) $94.0 \mathrm{GHz}$

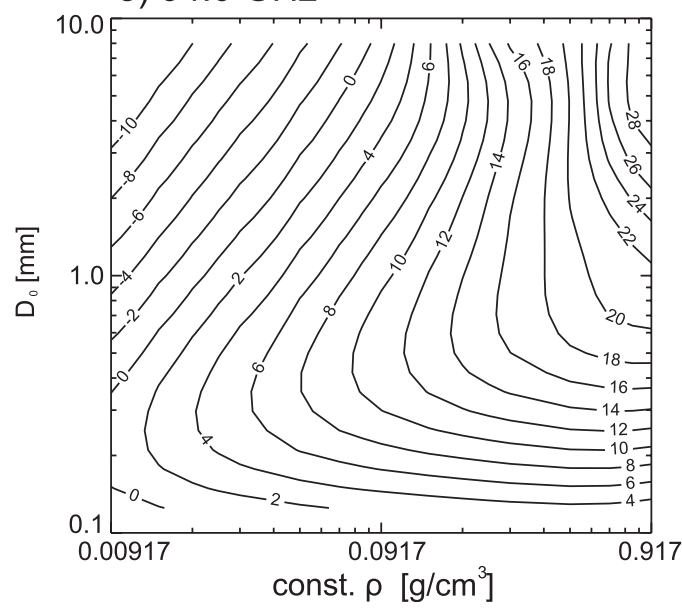

2-Way PIA [dB] per $1 \mathrm{~kg} / \mathrm{m}^{2}$ IWP

b) $13.4 \mathrm{GHz}$

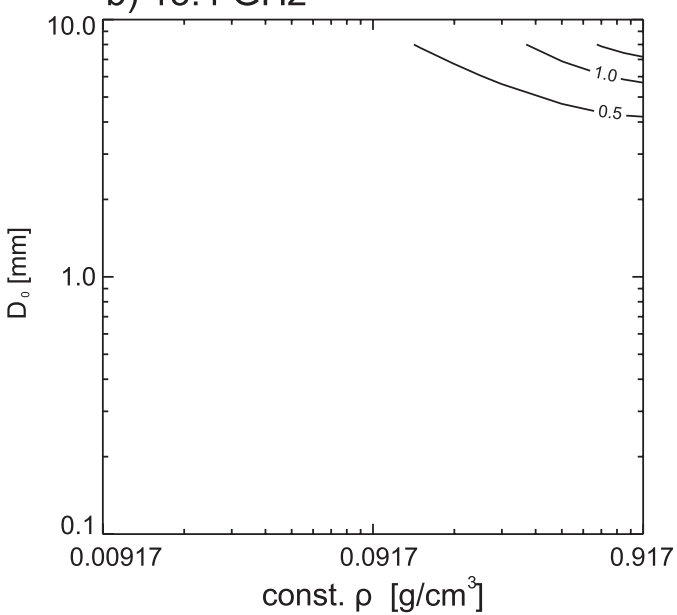

d) $35.6 \mathrm{GHz}$

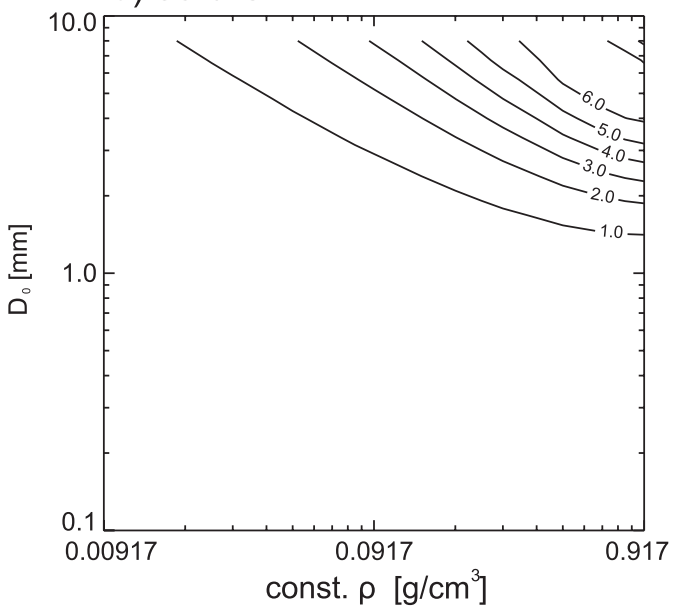

f) $94.0 \mathrm{GHz}$

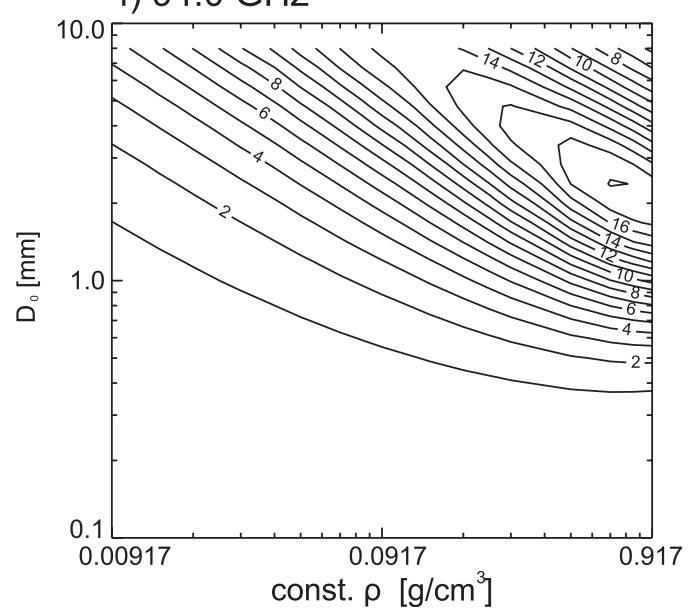

FIG. 8. (left) The effective reflectivity factor $Z_{e}$ for $\mathcal{W}=1 \mathrm{~g} \mathrm{~m}^{-3}$ and (right) two-way path attenuation per $1 \mathrm{~kg} \mathrm{~m}^{-2}$ of ice water path for $13.4-\mathrm{GHz}$ (a) reflectivity and (b) attenuation, 35.6-GHz (c) reflectivity and (d) attenuation, and 94.0-GHz (e) reflectivity and (f) attenuation. 


\section{$Z_{\text {eff }}[d B Z]$}

a) $13.4 \mathrm{GHz}$

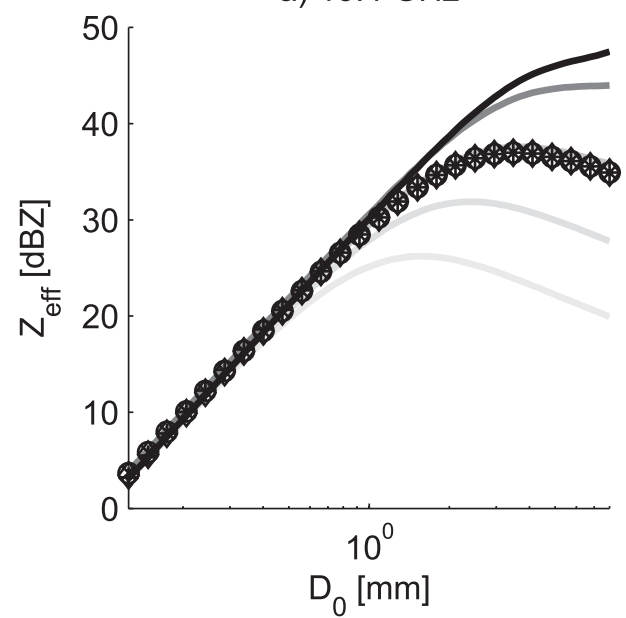

c) $35.6 \mathrm{GHz}$

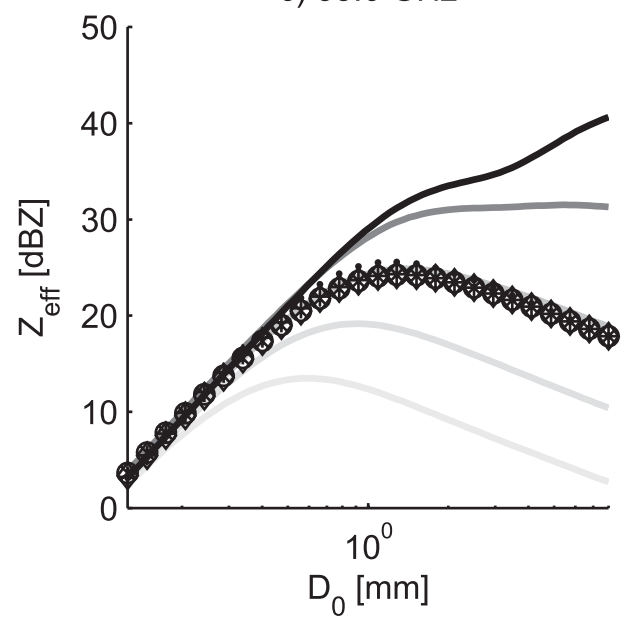

e) $94.0 \mathrm{GHz}$

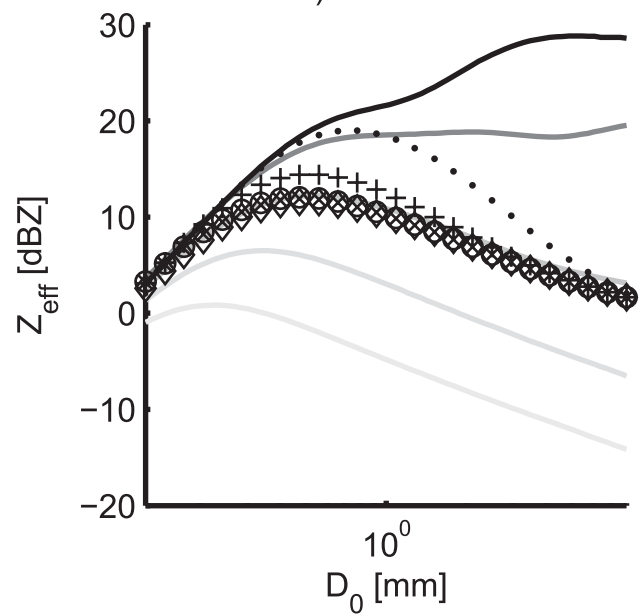

2-way PIA [dB] per $1 \mathrm{~kg} / \mathrm{m}^{\wedge} 2$ IWP

b) $13.4 \mathrm{GHz}$

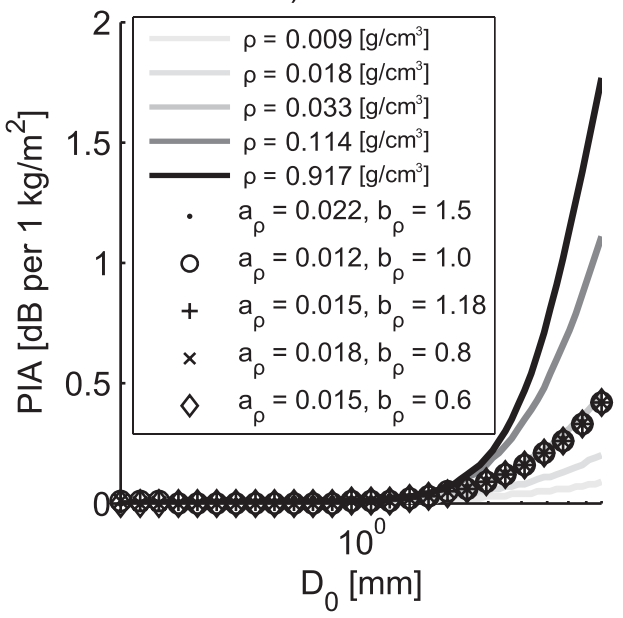

d) $35.6 \mathrm{GHz}$

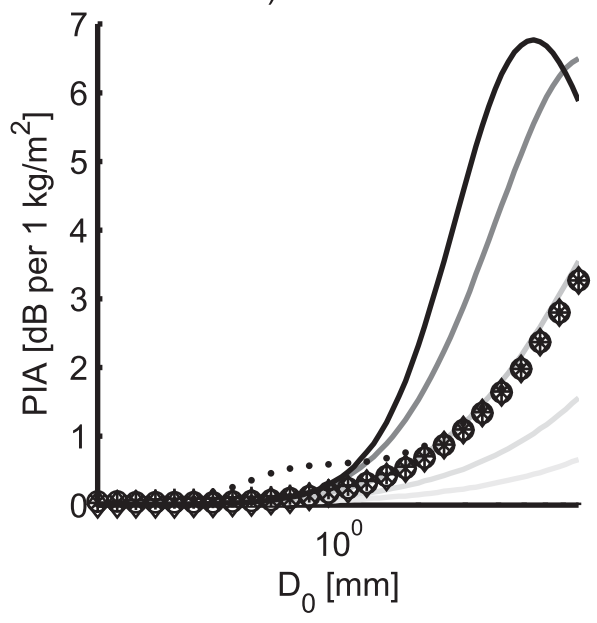

f) $94.0 \mathrm{GHz}$

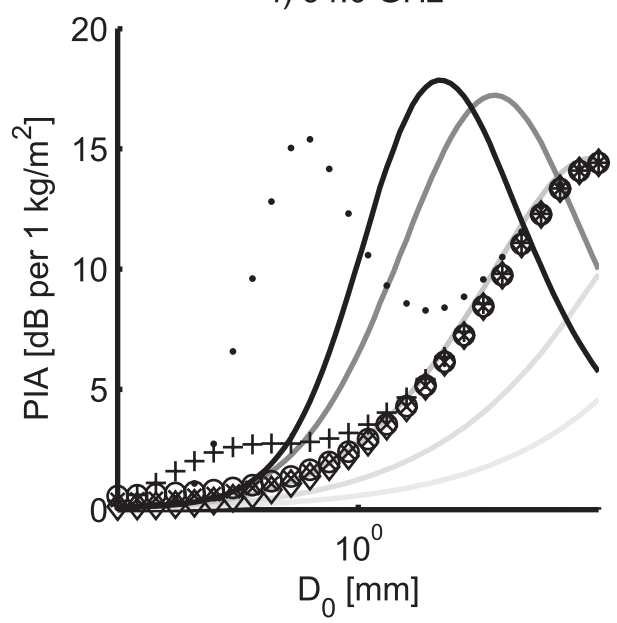

FIG. 9. Similar to Fig. 8, but including variable-density PSD-integrated reflectivities (points) and constantdensity PSD-integrated reflectivities (solid lines). 
to errors in either hydrometeor property. Moreover, two-way path attenuation per unit ice water path becomes large (up to $18 \mathrm{~dB}$ ) for dense ice particles with $D_{0}$ larger than a few hundred micrometers. Although we did not present results for the highest frequencies of the Advanced Microwave Sounding Unit $(183 \mathrm{GHz})$ or the GMI (166 GHz), our findings for $150 \mathrm{GHz}$ are broadly applicable to those frequencies as well.

In the interest of isolating the effects of variable ice particle properties, we did not consider the roles of cloud liquid water or gaseous absorption and emission on observed microwave brightness temperatures. It is understood that any additional absorber or attenuation in the atmospheric column will tend to reduce the overall sensitivity of microwave observables to parameter variations we have described herein; we therefore present these results as "worst case" sensitivities.

Although this study is theoretical in nature, it does lead to some recommendations to the broader remote sensing and validation communities who observe and measure ice-phase precipitation. The primary recommendation is to make direct measurements of the microwave extinction (and scattering, if possible) by hydrometeors at several frequencies consistent with those employed on current passive and active microwave remote sensing platforms. This should also include coincident measurements of the physical properties of size, shape, fall speed, and so on such that our physical assumptions can be closely linked to the measured extinction properties. Having an instrumentation suite at various ground validation sites that makes such measurements would be of significant benefit to reducing the above uncertainties.

Acknowledgments. We thank Dr. Robert Meneghini for his constructive comments on multiple-component dielectric mixing. We also thank an anonymous reviewer for useful recommendations. This project was funded through NASA Grants NAG7-7741 and NAG5-9894.

\section{REFERENCES}

Barthazy, E., W. Heinrich, and A. Waldvogel, 1998: Size distribution of hydrometers through the melting layer. Atmos. Res., 47-48, 193-208.

Battaglia, A., J. M. Haynes, T. L'Ecuyer, and C. Simmer, 2008: Identifying multiple-scattering-affected profiles in CloudSat observations over the oceans. J. Geophys. Res., 113, D00A17, doi:10.1029/2008JD009960.

Bohren, C. F., and D. R. Huffman, 1983: Absorption and Scattering of Light by Small Particles. Wiley-Interscience, $530 \mathrm{pp}$.

Brandes, E. A., G. Zhang, and J. Vivekanandan, 2004: Drop size distribution retrieval with polarimetric radar. Model Appl., 43, 461-475.

Brown, S. T., and C. S. Ruf, 2007: Validation and development of melting layer models using constraints by active/passive microwave observations of rain and the wind-roughened ocean surface. J. Atmos. Oceanic Technol., 24, 543-563.

Bruggeman, D. A. G., 1935: Berechnung verschiedener physikalischer Konstanten von heterogenen Substanzen. I. Dielektrizitatskonstanten und Leitfahigkeiten der Mischkörper aus isotropen Substanzen (Calculation of various physical constants of heterogeneous substances. I. Dielectric constants and conductivities of mixed matter from isotropic substances). Ann. Phys., 24, 636-679.

Casella, D., A. Mugnai, P. Sano, and M. Formenton, 2008: Microwave single-scattering properties of randomly oriented soft-ice hydrometeors. Adv. Geosci., 17, 79-85.

Donovan, D., M. Quante, I. Schlimme, and A. Macke, 2004: Use of equivalent spheres to model the relation between radar reflectivity and optical extinction of ice cloud particles. Appl. Opt., 43, 4929-4940.

Han, M., S. A. Braun, W. S. Olson, P. O. G. Persson, and J.-W. Bao, 2010: Application of the TRMM PR and TMI measurements to assess cloud microphysical schemes in the MM5 model for a winter storm. J. Appl. Meteor. Climatol., 49, 1129-1148.

Hashino, T., and G. J. Tripoli, 2007: The Spectral Ice Habit Prediction System (SHIPS). Part I: Model description and simulation of the vapor deposition process. J. Atmos. Sci., 64, 2210-2237.

Heymsfield, A. J., 2003: Properties of tropical and midlatitude ice cloud particle ensembles. Part II: Applications for mesoscale and climate models. J. Atmos. Sci., 60, 2592-2611.

_ Z. Z. Wang, and S. Y. Matrosov, 2005: Improved radar ice water content retrieval algorithms using coincident microphysical and radar measurements. J. Appl. Meteor., 44, 1391-1412.

Hogan, R. J., L. Tian, P. R. A. Brown, C. D. Westbrook, A. J. Heymsfield, and J. D. Eastment, 2012: Radar scattering from ice aggregates using the horizontally aligned oblate spheroid approximation. J. Appl. Meteor. Climatol., 51, 655-671.

Hong, G., 2007a: Parameterization of scattering and absorption properties of nonspherical ice crystals at microwave frequencies. J. Geophys. Res., 112, D11208, doi:10.1029/2006JD008364.

_ 2007b: Radar backscattering properties of nonspherical ice crystals at 94 GHz. J. Geophys. Res., 112, D22203, doi:10.1029/ 2007JD008839.

Ishimoto, H., 2008: Radar backscattering computations for fractalshaped snowflakes. J. Meteor. Soc. Japan, 86, 459-469.

Katsumata, M., H. Uyeda, K. Iwanami, and G. Liu, 2000: The response of 36- and 89-GHz microwave channels to convective snow clouds over ocean: Observation and modeling. J. Appl. Meteor., 39, 2322-2335.

Kim, M.-J., M. S. Kulie, C. O'Dell, and R. Bennartz, 2007: Scattering of ice particles at microwave frequencies: A physically based parameterization. J. Appl. Meteor. Climatol., 46, 615-633.

— J. A. Weinman, W. S. Olson, D.-E. Chang, G. SkofronickJackson, and J. Wang, 2008: A physical model to estimate snowfall over land using AMSU-B observations. J. Geophys. Res., 113, D09201, doi:10.1029/2007JD008589.

Kulie, M. S., and R. Bennartz, 2009: Utilizing spaceborne radars to retrieve dry snowfall. J. Appl. Meteor. Climatol., 48, 2564 2580.

— — - T. J. Greenwald, Y. Chen, and F. Weng, 2010: Uncertainties in microwave properties of frozen precipitation: Implications for remote sensing and data assimilation. $J$. Atmos. Sci., 67, 3471-3487.

Kummerow, C., W. S. Olson, and L. Giglio, 1996: A simplified scheme for obtaining precipitation and vertical hydrometeor profiles from passive microwave sensors. IEEE Trans. Geosci. Remote Sens., 34, 1213-1232. 
Lakhtakia, A., and B. Shanker, 1993: Beltrami fields within continuous source regions, volume integral equations, scattering algorithms, and the extended Maxwell-Garnett model. Int. J. Appl. Electromag. Mater., 4, 65-82.

Lenoble, J., 1985: Radiative Transfer in Scattering and Absorbing Atmospheres: Standard Computational Procedures. A. Deepak, $300 \mathrm{pp}$.

Liou, K. N., 2002: An Introduction to Atmospheric Radiation. 2nd ed. Int. Geophys. Series, Vol. 84, Academic Press, 583 pp.

Liu, G., 2008: A database of microwave single-scattering properties for nonspherical ice particles. Bull. Amer. Meteor. Soc., 89, $1563-1570$.

Liu, Q., and F. Weng, 2002: A microwave polarimetric two-stream radiative transfer model. J. Atmos. Sci., 59, 2396-2402.

Locatelli, J., and P. Hobbs, 1974: Fall speeds and masses of solid precipitation particles. J. Geophys. Res., 98, 2757-2765.

Mackay, T. G., 2005: Linear and nonlinear homogenized composite mediums as metamaterials. Electromagnetics, 25, 461-481.

Magono, C., and T. Nakamura, 1965: Aerodynamic studies of falling snowflakes. J. Meteor. Soc. Japan, 43, 139-147.

Matrosov, S. Y., 1998: A dual-wavelength radar method to measure snowfall rate. J. Appl. Meteor., 37, 1510-1521.

— A. J. Heymsfield, and Z. Wang, 2005: Dual-frequency radar ratio of nonspherical atmospheric hydrometeors. Geophys. Res. Lett., 32, L13816, doi:10.1029/2005GL023210.

_ - A. Battaglia, and P. Rodriguez, 2008: Effects of multiple scattering on attenuation-based retrievals of stratiform rainfall from CloudSat. J. Atmos. Oceanic Technol., 25, 2199-2208.

Maxwell Garnett, J. C., 1904: Colours in metal glasses and in metallic films. Philos. Trans. Roy. Soc., 203, 385-420.

Meneghini, R., and L. Liao, 2000: Effective dielectric constants of mixed-phase hydrometeors. J. Atmos. Oceanic Technol., 17, 628-639.

Mie, G., 1908: Beiträge zur Optik trüber Medien, speziell kolloidaler Metallösungen (Contributions to the optics of turbid media, specifically colloidal metal solutions). Ann. Phys., 26, 597-614.

Mitchell, D. L., R. Zhang, and R. L. Pitter, 1990: Mass-dimensional relationships for ice particles and the influence and riming on snowfall rates. J. Appl. Meteor., 29, 153-163.

Noh, Y.-J., G. Liu, E.-K. Seo, and J. R. Wang, 2006: Development of a snowfall retrieval algorithm at high microwave frequencies. J. Geophys. Res., 111, D22216, doi:10.1029/2005JD006826.

Panegrossi, G., and Coauthors, 1998: Use of cloud model microphysics for passive microwave-based precipitation retrieval: Significance of consistency between model and measurement manifolds. J. Atmos. Sci., 55, 1644-1673.

Petty, G. W., 2001: Physical and microwave radiative properties of precipitating clouds. Part I: Principal component analysis of observed multichannel microwave radiances in tropical stratiform rainfall. J. Appl. Meteor., 40, 2105-2114.
- 2006: A First Course in Atmospheric Radiation. Sundog Publishing, $460 \mathrm{pp}$.

— , and W. Huang, 2010: Microwave backscatter and extinction by soft ice spheres and complex snow aggregates. J. Atmos. Sci., 67, 769-787.

— inhomogeneous and nonspherical particles: Key relationships and conversions. J. Atmos. Sci., 68, 1460-1473.

Savage, R. C., 1978: The radiative properties of hydrometeors at microwave frequencies. J. Appl. Meteor., 17, 904.

Sihvola, A. H., and J. A. Kong, 1988: Effective permittivity of dielectric mixtures. IEEE Trans. Geosci. Remote Sens., 26, 420-429.

Skofronick-Jackson, G. M., A. Heymsfield, E. Holthaus, C. Albers, and M.-J. Kim, 2008: Nonspherical and spherical characterization of ice in Hurricane Erin for wideband passive microwave comparisons. J. Geophys. Res., 113, D06201, doi:10.1029/ 2007JD008866.

Smith, E. A., A. Mugnai, H. J. Cooper, G. J. Tripoli, and X. Xiang, 1992: Foundations for statistical-physical precipitation retrieval from passive microwave satellite measurements. Part I: Brightness-temperature properties of a time-dependent cloud-radiation model. J. Appl. Meteor., 31, 506-531.

Surussavadee, C., and D. H. Staelin, 2006: Comparison of AMSU millimeter-wave satellite observations, MM5/TBSCAT predicted radiances, and electromagnetic models for hydrometeors. IEEE Trans. Geosci. Remote Sens., 44, 2667-2678.

Szyrmer, W., and I. Zawadzki, 2010: Snow studies. Part II: Average relationship between mass of snowflakes and their terminal fall velocity. J. Atmos. Sci., 67, 3319-3335.

Tripoli, G. J., 1992: A nonhydrostatic mesoscale model designed to simulate scale interaction. Mon. Wea. Rev., 120, 1342-1359.

Ulbrich, C. W., 1983: Natural variations in the analytical form of the raindrop size distribution. J. Climate Appl. Meteor., 22, 1764-1775.

— erties: Analysis methods for drop size spectra. J. Appl. Meteor., 37, 912-923.

Warren, S., 1984: Optical constants of ice from the ultraviolet to the microwave. Appl. Opt., 23, 1206-1225.

- , and R. Brandt, 2008: Optical constants of ice from the ultraviolet to the microwave: A revised compilation. J. Geophys. Res., 113, D14220, doi:10.1029/2007JD009744.

Weng, F., and N. Grody, 2000: Retrieval of ice cloud parameters using a microwave imaging radiometer. J. Atmos. Sci., 57, 1069-1081.

Wu, L., and G. Petty, 2010: Intercomparison of bulk microphysics schemes in model simulations of polar lows. Mon. Wea. Rev., 138, 2211-2228.

Zhao, L. M., and F. Weng, 2002: Retrieval of ice cloud parameters using the Advanced Microwave Sounding Unit. J. Appl. Meteor., 41, 384-395. 